\title{
An introduction to the topological derivative
}

\author{
S. Amstutz \\ CMAP - Ecole polytechnique \\ Route de Saclay, 91120 Palaiseau, France
}

\begin{abstract}
Purpose. This paper provides a self-contained introduction to the mathematical aspects of the topological derivative.

Design / methodology / approach. Full justifications are given on simple model problems following a modern approach based on the averaged adjoint state technique. Extensions are discussed in relation with the literature on the field.

Findings. Closed expressions of topological derivatives are obtained and commented.

Originality / value. Several cases are covered in a unified and didactic presentation. Some elements of proof are novel.
\end{abstract}

\section{Introduction}

Let $D$ be a bounded, open subset of $\mathbb{R}^{N}$, the "hold-all" domain. With applications to shape optimization in mind, it is mostly assumed that $N=2$ or $N=3$, but the case $N=1$ may have its own interest, and the cases $N>3$ may be considered for mathematical generality. To fix ideas let $\mathcal{A}=\{\Omega \subset D, \Omega$ open $\}$, and consider a function

$$
\Omega \in \mathcal{A} \mapsto \mathcal{J}(\Omega) \in \mathbb{R}
$$

indifferently called shape functional, or cost function, or criterion. The concept of topological derivative was formally introduced in [Eschenauer et al., 1994, Schumacher, 1995] as the "bubble method" for the optimal design of structures, and the first mathematical justifications appeared in [Sokolowski and Żochowski, 1999, Garreau et al., 2001]. It can be defined as follows.

Definition 1.1 Let $\omega$ be a bounded, open subset of $\mathbb{R}^{N}$. We say that $\mathcal{J}$ admits a topological derivative at $\Omega_{0} \in \mathcal{A}$ and at the point $z \in \Omega_{0}$ with respect to $\omega$ if there exists a function $f: \mathbb{R}_{+} \rightarrow \mathbb{R}_{+}$with $\lim _{\varepsilon \searrow 0} f(\varepsilon)=0$ such that the following limit exists:

$$
d_{T} \mathcal{J}\left(\Omega_{0}, \omega, z\right)=\lim _{\varepsilon \searrow 0} \frac{\mathcal{J}\left(\Omega_{0} \backslash(\overline{z+\varepsilon \omega})\right)-\mathcal{J}\left(\Omega_{0}\right)}{f(\varepsilon)} .
$$

Of course, (1) is equivalent to the "topological asymptotic expansion":

$$
\mathcal{J}\left(\Omega_{0} \backslash(\overline{z+\varepsilon \omega})\right)-\mathcal{J}\left(\Omega_{0}\right)=f(\varepsilon) d_{T} \mathcal{J}\left(\Omega_{0}, \omega, z\right)+o(f(\varepsilon)) .
$$

The set $\omega_{\varepsilon}:=z+\varepsilon \omega$ is the domain perturbation, see Figure 1. It is often called hole, or cavity, or more specifically inclusion when it is filled with a material phase different from the background one. The set $\Omega_{\varepsilon}:=\Omega_{0} \backslash \bar{\omega}_{\varepsilon}$ is the perturbed or perforated domain. The set $\omega$ is the perturbation at scale 
1. It is typically chosen as the unit ball, but other cases, like cracks, can be of interest. Clearly, for the volume functional $\mathcal{J}(\Omega)=|\Omega|$, we have $d_{T} \mathcal{J}(\Omega, \omega, z)=-1$ for all $z \in \Omega$ with $f(\varepsilon)=\left|\omega_{\varepsilon}\right|=\varepsilon^{N}|\omega|$. The perimeter functional $\mathcal{J}(\Omega)=|\partial \Omega|$ yields $d_{T} \mathcal{J}(\Omega, \omega, z)=1$ for all $z \in \Omega$ with $f(\varepsilon)=\varepsilon^{N-1}|\partial \omega|$.

In most practical cases the shape functional involves the solution of a boundary value problem, the so-called (direct) state. Then the type of boundary condition around the hole has a drastic effect on its asymptotic behavior. One case turns out to be much easier to analyze than the others: it is when the perturbation is on coefficients of non-principal parts of the differential operator. This situation is studied first, while the main content of this presentation concerns perturbations of the principal part in the form of transmission, Neumann and Dirichlet conditions. In this introductory paper we devote to provide a rigorous mathematical analysis of the aforementioned issues on simple model problems. In particular we restrict ourselves to Laplace-type elliptic boundary value problems and simple shape functionals. To ease the reading, the use of boundary integral equations is bypassed, and non-standard function spaces are described in some details as soon as they are needed. Extensions and refinements are discussed in comments, in connection with the related literature.

From the technical standpoint, a crucial aspect of the topological sensitivity analysis is the incorporation of the adjoint state while we are typically not working in a differentiable framework. This is in contrast with smooth shape variations and the more classical notion of shape derivative [Murat and Simon, 1976, Sokolowski and Zolésio, 1992, Allaire, 2007, Henrot and Pierre, 2018]. Several approaches have been developed to overcome this issue, including the domain truncation method [Masmoudi, 2002, Garreau et al., 2001, Guillaume and Sid Idris, 2002, Novotny et al., 2019a, Novotny and Sokolowski, 2020], the generalized Lagrangian method [Amstutz, 2003, Amstutz, 2006a, Amstutz, 2006b, Amstutz and Dominguez, 2008], and the topological shape sensitivity method [Novotny, 2003, Novotny and Sokolowski, 2013]. Other authors only incorporate the adjoint state in the end of the derivations [Guzina and Bonnet, 2006, Bonnet and Delgado, 2013]. Here we will follow the averaged adjoint method recently introduced in [Delfour and Sturm, 2017] in an abstract setting and in [Sturm, 2020, Gangl and Sturm, 2020] in this context, which is a modification of the generalized Lagrangian method. This formulation, initially mainly developed for nonlinear purposes, turns out to be somehow natural. It is also elegant in the linear context as it expresses the variation of the cost function exactly as the variation of the Lagrangian.

The paper is organized as follows. Section 2 is dedicated to the perturbation of non-principal parts, where differential calculus and the standard adjoint state method can be used. The averaged adjoint method is presented section 3 in an abstract linear framework. Section 4 deals with the cases of transmission and Neumann conditions in dimension $N \geq 1$. Sections 5 and 6 address the Dirichlet case in $2 \mathrm{D}$ and in $3 \mathrm{D}$, respectively, as here these two space dimensions need to be distinguished and the $1 \mathrm{D}$ case is irrelevant.

\section{Perturbation of non-principal parts}

In this section we specifically assume that $N \in\{1,2,3\}$, although higher dimensions could be addressed upon minor adaptations. A key element of the analysis will be the Sobolev embedding $H_{0}^{1}(D) \hookrightarrow L^{p}(D)$ with $p$ recalled in Table 1, see e.g. [Brezis, 2011, Demengel and Demengel, 2012, Attouch et al., 2014]. For any measurable set $\Omega \subset D$ we consider the boundary value problem

$$
\left\{\begin{array}{l}
-\Delta u_{\Omega}+h_{\Omega} u_{\Omega}=f_{\Omega} \text { in } D \\
u_{\Omega}=0 \text { on } \partial D,
\end{array}\right.
$$

where the functions $h_{\Omega}$ and $f_{\Omega}$ are defined using the characteristic function $\chi_{\Omega}$ of $\Omega$ by

$$
h_{\Omega}=\chi_{\Omega} h_{1}+\left(1-\chi_{\Omega}\right) h_{0}, \quad f_{\Omega}=\chi_{\Omega} f_{1}+\left(1-\chi_{\Omega}\right) f_{0} .
$$




\section{$\omega_{\bullet}^{*} \cdot$}

$\Omega_{\varepsilon}$

Figure 1: Domain perturbation.

The above functions $h_{0}, h_{1} \in L^{q}(D), f_{0}, f_{1} \in L^{r}(D)$ are supposed to be given, with $h_{0}, h_{1} \geq 0$ and $q, r$ as in Table 1 . Finally we consider a shape functional of the form $\mathcal{J}(\Omega)=J\left(u_{\Omega}\right)$ where $J: H_{0}^{1}(D) \rightarrow \mathbb{R}$ is of class $\mathcal{C}^{2}$.

\begin{tabular}{|c|c|c|c|}
\hline $\mathrm{N}$ & $\mathrm{p}$ & $\mathrm{q}$ & $\mathrm{r}$ \\
\hline 1 & $\leq+\infty$ & $>2$ & $>2$ \\
\hline 2 & $<+\infty$ & $>2$ & $>2$ \\
\hline 3 & $\leq 6$ & $>6$ & $>3$ \\
\hline
\end{tabular}

Table 1: Lebesgue exponents

The following result extends ideas from [Hassine et al., 2007], see also [Novotny and Sokolowski, 2013].

Theorem 2.1 The above shape functional admits at a.e. $z \in \Omega$ the topological derivative

$$
d_{T} \mathcal{J}(\Omega, \omega, z)=\left[\left(h_{0}-h_{1}\right) u_{\Omega} v_{\Omega}-\left(f_{0}-f_{1}\right) v_{\Omega}\right](z)
$$

with $f(z)=\varepsilon^{N}|\omega|$, where the adjoint state $v_{\Omega}$ is the solution of

$$
\int_{D}\left(\nabla v_{\Omega} \cdot \nabla \varphi+h_{\Omega} v_{\Omega} \varphi\right) d x=-d J\left(u_{\Omega}\right) \varphi d x \quad \forall \varphi \in H_{0}^{1}(D)
$$

Proof. Given $p>2$ as in Table 1 we define, for all $(u, v, h, f) \in H_{0}^{1}(D) \times H_{0}^{1}(D) \times L^{p /(p-2)}(D) \times$ $L^{p /(p-1)}(D)$, the quantities

$$
a(h, u, v)=\int_{D}(\nabla u \cdot \nabla v+h u v) d x, \quad l(f, v)=\int_{D} f v d x
$$

These expressions are well defined since $u v \in L^{p / 2}(D)$ by the Cauchy-Schwarz inequality. For any $(h, f) \in L^{p /(p-2)}(D) \times L^{p /(p-1)}(D)$ we define $A(h) \in \mathcal{L}\left(H_{0}^{1}(D), H^{-1}(D)\right)$ and $L(f) \in H^{-1}(D)$ by

$$
\langle A(h) u, v\rangle_{H^{-1}(D), H_{0}^{1}(D)}=a(h, u, v), \quad\langle L(f), v\rangle_{H^{-1}(D), H_{0}^{1}(D)}=l(f, v) .
$$

Set

$$
\mathcal{U}=\left\{(h, f) \in L^{p /(p-2)}(D) \times L^{p /(p-1)}(D), h \geq 0 \text { a.e. }\right\}
$$


By the Lax-Milgram theorem and the Poincaré inequality we have $A(h) \in \operatorname{isom}\left(H_{0}^{1}(D), H^{-1}(D)\right)$ for every $(h, f)$ in a neighborhood of any $\left(f_{0}, h_{0}\right) \in \mathcal{U}$. In addition, due to its affine structure, the map $(h, f) \in L^{p /(p-2)}(D) \times L^{p /(p-1)}(D) \mapsto(A(h), L(f))$ is of class $\mathcal{C}^{\infty}$. It follows that the map $(h, f) \mapsto u(h, f):=A(h)^{-1} L(f)$ is of class $\mathcal{C}^{\infty}$ in a neighborhood of any element of $\mathcal{U}$. In order to find a convenient expression of the derivative we use the classical adjoint method, based on the Lagrangian

$$
\mathcal{L}(h, f, u, v)=J(u)+a(h, u, v)-l(f, v) .
$$

We differentiate $j(h, f):=J(u(h, f))=\mathcal{L}(h, f, u(h, f), v)$ at $\left(h_{\Omega}, f_{\Omega}\right)$ for an arbitrary $v \in H_{0}^{1}(D)$ :

$$
d j\left(h_{\Omega}, f_{\Omega}\right)(\hat{u}, \hat{f})=d_{h} \mathcal{L}\left(h_{\Omega}, f_{\Omega}, u_{\Omega}, v\right) \hat{h}+d_{f} \mathcal{L}\left(h_{\Omega}, f_{\Omega}, u_{\Omega}, v\right) \hat{f}+d_{u} \mathcal{L}\left(h_{\Omega}, f_{\Omega}, u_{\Omega}, v\right) d u\left(h_{\Omega}, f_{\Omega}\right)(\hat{h}, \hat{f}) .
$$

Choosing $v$ as the adjoint state $v_{\Omega}$ cancels the last term by construction. We arrive at

$$
\operatorname{dj}\left(h_{\Omega}, f_{\Omega}\right)(\hat{u}, \hat{f})=\int_{D} \hat{h} u_{\Omega} v_{\Omega} d x-\int_{D} \hat{f} v_{\Omega} d x
$$

By Taylor-Lagrange expansion this implies

$$
j\left(h_{\Omega}+\hat{h}, f_{\Omega}+\hat{f}\right)-j\left(h_{\Omega}, f_{\Omega}\right)=\int_{D} \hat{h} u_{\Omega} v_{\Omega} d x-\int_{D} \hat{f} v_{\Omega} d x+O\left(\|\hat{h}\|_{L^{p /(p-2)}(D)}^{2}+\|\hat{f}\|_{L^{p /(p-1)}(D)}^{2}\right) .
$$

Choose $z \in \Omega$ and $\varepsilon$ small enough so that

$$
\hat{h}:=h_{\Omega \backslash(\overline{z+\varepsilon \omega})}-h_{\Omega}=\chi_{z+\varepsilon \omega}\left(h_{0}-h_{1}\right), \quad \hat{f}:=f_{\Omega \backslash(\overline{z+\varepsilon \omega})}-f_{\Omega}=\chi_{z+\varepsilon \omega}\left(f_{0}-f_{1}\right) .
$$

It is straightforward from Hölder's inequality that

$$
\begin{aligned}
\|\hat{h}\|_{L^{p /(p-2)}(D)} & \leq\left(\varepsilon^{N}|\omega|\right)^{1-\frac{2}{p}-\frac{1}{q}}\left\|h_{0}-h_{1}\right\|_{L^{q}(D)} \\
\|\hat{f}\|_{L^{p /(p-1)}(D)} & \leq\left(\varepsilon^{N}|\omega|\right)^{1-\frac{1}{p}-\frac{1}{r}}\left\|f_{0}-f_{1}\right\|_{L^{r}(D)} .
\end{aligned}
$$

With the assumptions made on $q$ and $r$ we can adjust $p$ in accordance with Table 1 in order to have

$$
\|\hat{h}\|_{L^{p /(p-2)}(D)}^{2}+\|\hat{f}\|_{L^{p /(p-1)}(D)}^{2}=o\left(\varepsilon^{N}\right)
$$

So far we have shown that

$$
\mathcal{J}(\Omega \backslash(\overline{z+\varepsilon \omega}))-\mathcal{J}(\Omega)=\int_{z+\varepsilon \omega}\left(\left(h_{0}-h_{1}\right) u_{\Omega} v_{\Omega}-\left(f_{0}-f_{1}\right) v_{\Omega}\right) d x+o\left(\varepsilon^{N}\right) .
$$

This can be rewritten as

$$
\begin{aligned}
\mathcal{J}(\Omega & \backslash(\overline{z+\varepsilon \omega}))-\mathcal{J}(\Omega)=|\omega| \varepsilon^{N}\left[\left(h_{0}-h_{1}\right) u_{\Omega} v_{\Omega}-\left(f_{0}-f_{1}\right) v_{\Omega}\right](z) \\
& +\int_{z+\varepsilon \omega}\left(\left(\left(h_{0}-h_{1}\right) u_{\Omega} v_{\Omega}-\left(f_{0}-f_{1}\right) v_{\Omega}\right)(x)-\left(\left(h_{0}-h_{1}\right) u_{\Omega} v_{\Omega}-\left(f_{0}-f_{1}\right) v_{\Omega}\right)(z)\right) d x+o\left(\varepsilon^{N}\right)
\end{aligned}
$$

By Lebesgue's differentiation theorem this latter integral is a $o\left(\varepsilon^{N}\right)$ for a.e. $z \in \Omega$.

We observe here that the topological derivative does not depend on the shape of $\omega$. We will see that this property is usually lost when principal parts are perturbed. 


\section{A generalized adjoint method}

In the previous section we have been able to use Fréchet's differential calculus and the classical Lagrangian method thanks to Sobolev embeddings and the fact that the characteristic function of the hole was small in an appropriate $L^{p}$ norm. When we perturb the principal part of the differential operator, differential calculus applies in $L^{\infty}$, but the $L^{\infty}$ norm of the characteristic function of a set of positive Lebesgue measure does not go to zero with the diameter of the set. As explained in the introduction we are going to use a generalization of the Lagrangian framework based on the averaged adjoint state idea from [Delfour and Sturm, 2017, Sturm, 2020, Gangl and Sturm, 2020].

Proposition 3.1 Let $H$ be a Hilbert space and $\varepsilon_{0}>0$. For every $\varepsilon \in\left[0, \varepsilon_{0}\right]$ we consider :

- a bilinear form $a_{\varepsilon}(\cdot, \cdot)$ on $H$,

- a linear form $l_{\varepsilon}(\cdot)$ on $H$,

- $a$ direct state $u_{\varepsilon} \in H$ solution of

$$
a_{\varepsilon}\left(u_{\varepsilon}, \varphi\right)=l_{\varepsilon}(\varphi) \quad \forall \varphi \in H,
$$

- a cost function $J_{\varepsilon}(\cdot)$ continuously Fréchet differentiable on $H$,

- an adjoint state $v_{\varepsilon} \in H$ solution of

$$
a_{\varepsilon}\left(\varphi, v_{\varepsilon}\right)=-\int_{0}^{1} d J_{\varepsilon}\left(t u_{\varepsilon}+(1-t) u_{0}\right) \varphi d t \quad \forall \varphi \in H .
$$

Then we have for all $\varepsilon \in\left[0, \varepsilon_{0}\right]$

$$
J_{\varepsilon}\left(u_{\varepsilon}\right)-J_{0}\left(u_{0}\right)=\left(\mathcal{L}_{\varepsilon}-\mathcal{L}_{0}\right)\left(u_{0}, v_{\varepsilon}\right),
$$

with the Lagrangian

$$
\mathcal{L}_{\varepsilon}(u, v)=J_{\varepsilon}(u)+a_{\varepsilon}(u, v)-l_{\varepsilon}(v) \quad \forall(\varepsilon, u, v) \in\left[0, \varepsilon_{0}\right] \times H \times H .
$$

ProOF. We have the easy equalities:

$$
\begin{aligned}
J_{\varepsilon}\left(u_{\varepsilon}\right)-J_{0}\left(u_{0}\right) & =\mathcal{L}_{\varepsilon}\left(u_{\varepsilon}, v_{\varepsilon}\right)-\mathcal{L}_{0}\left(u_{0}, v_{\varepsilon}\right) \\
& =\mathcal{L}_{\varepsilon}\left(u_{\varepsilon}, v_{\varepsilon}\right)-\mathcal{L}_{\varepsilon}\left(u_{0}, v_{\varepsilon}\right)+\mathcal{L}_{\varepsilon}\left(u_{0}, v_{\varepsilon}\right)-\mathcal{L}_{0}\left(u_{0}, v_{\varepsilon}\right) \\
& =J_{\varepsilon}\left(u_{\varepsilon}\right)+a_{\varepsilon}\left(u_{\varepsilon}, v_{\varepsilon}\right)-J_{\varepsilon}\left(u_{0}\right)-a_{\varepsilon}\left(u_{0}, v_{\varepsilon}\right)+\mathcal{L}_{\varepsilon}\left(u_{0}, v_{\varepsilon}\right)-\mathcal{L}_{0}\left(u_{0}, v_{\varepsilon}\right) \\
& =J_{\varepsilon}\left(u_{\varepsilon}\right)-J_{\varepsilon}\left(u_{0}\right)+a_{\varepsilon}\left(u_{\varepsilon}-u_{0}, v_{\varepsilon}\right)+\mathcal{L}_{\varepsilon}\left(u_{0}, v_{\varepsilon}\right)-\mathcal{L}_{0}\left(u_{0}, v_{\varepsilon}\right) \\
& =J_{\varepsilon}\left(u_{\varepsilon}\right)-J_{\varepsilon}\left(u_{0}\right)-\int_{0}^{1} d J_{\varepsilon}\left(t u_{\varepsilon}+(1-t) u_{0}\right)\left(u_{\varepsilon}-u_{0}\right) d t+\mathcal{L}_{\varepsilon}\left(u_{0}, v_{\varepsilon}\right)-\mathcal{L}_{0}\left(u_{0}, v_{\varepsilon}\right) .
\end{aligned}
$$

The first three terms cancel out due to

$$
d J_{\varepsilon}\left(t u_{\varepsilon}+(1-t) u_{0}\right)\left(u_{\varepsilon}-u_{0}\right)=\frac{d}{d t}\left[J_{\varepsilon}\left(t u_{\varepsilon}+(1-t) u_{0}\right)\right],
$$

which leads to the claim.

We stress that the variation of the Lagrangian needs to be evaluated at the variable adjoint state $v_{\varepsilon}$. We will see that in case of topology perturbations, approximating $v_{\varepsilon}$ by $v_{0}$ usually yields an error of first order. Note that $v_{0}$ is the standard unperturbed adjoint state, solution of

$$
a_{0}\left(\varphi, v_{0}\right)=-d J\left(u_{0}\right) \varphi \quad \forall \varphi \in H .
$$




\section{Inclusion and Neumann cases}

\subsection{Problem formulation}

Let $\Omega$ be an open and bounded subset of $\mathbb{R}^{N}, N \in \mathbb{N}^{*}$, and $\omega$ be a bounded, smooth open subset of $\mathbb{R}^{N}$. We consider a point $z \in \Omega$ and, for $\varepsilon \geq 0$ small enough, the set

$$
\omega_{\varepsilon}=z+\varepsilon \omega \subset \Omega \text {. }
$$

We recall the notation $\Omega_{\varepsilon}=\Omega \backslash \bar{\omega}_{\varepsilon}$, see Fig. 1 . We focus our attention on the problem

$$
\text { find } u_{\varepsilon} \in H_{0}^{1}(\Omega) \quad \text { s.t. } \int_{\Omega} \sigma_{\varepsilon} \nabla u_{\varepsilon} \cdot \nabla \varphi d x=\int_{\Omega} f \varphi d x \quad \forall \varphi \in H_{0}^{1}(\Omega),
$$

with $f \in L^{2}(\Omega)$ and the piecewise constant conductivity

$$
\sigma_{\varepsilon}=\chi_{\Omega_{\varepsilon}} \alpha+\chi_{\omega_{\varepsilon}} \beta, \quad \alpha>0, \beta \geq 0 .
$$

We will distinguish the two cases:

- $\beta>0$, called the inclusion case,

- $\beta=0$, called the Neumann case.

For simplicity, and to make the same setting applicable to the two above cases, we assume that $f=0$ in a neighborhood $z$, and that $\varepsilon$ is small enough so that $f=0$ in $\omega_{\varepsilon}$.

The inclusion case obviously admits a unique solution, and it corresponds to the strong form

$$
\begin{cases}-\operatorname{div}\left(\sigma_{\varepsilon} \nabla u_{\varepsilon}\right)=f & \text { in } \Omega \\ u_{\varepsilon}=0 & \text { on } \partial \Omega\end{cases}
$$

The Neumann case can be equivalently reformulated as

$$
\text { find } u_{\varepsilon} \in H_{0}^{1}(\Omega) \quad \text { s.t. } \int_{\Omega_{\varepsilon}} \alpha \nabla u_{\varepsilon} \cdot \nabla \varphi d x=\int_{\Omega_{\varepsilon}} f \varphi d x \quad \forall \varphi \in H_{0}^{1}(\Omega) \text {. }
$$

Since every function in $H^{1}\left(\Omega_{\varepsilon}\right)$ can be extended to a function in $H^{1}(\Omega)$, we recognize that $u_{\varepsilon \mid \Omega_{\varepsilon}}$ is the weak solution of

$$
\begin{cases}-\alpha \Delta u_{\varepsilon}=f & \text { in } \Omega_{\varepsilon} \\ \frac{\partial u_{\varepsilon}}{\partial n}=0 & \text { on } \partial \omega_{\varepsilon} \\ u_{\varepsilon}=0 & \text { on } \partial \Omega .\end{cases}
$$

In this case $u_{\varepsilon}$ is undefined inside $\omega_{\varepsilon}$. The "Neumann" terminology, of course, refers to the boundary condition on the hole. The boundary condition on $\partial \Omega$ plays no significant role in the present study.

In the two cases, $(2)$ corresponds for $\varepsilon=0$ to the unperturbed problem

$$
\begin{cases}-\alpha \Delta u_{0}=f & \text { in } \Omega \\ u_{0}=0 & \text { on } \partial \Omega .\end{cases}
$$

For consistence with the notation of Proposition 3.1 we set

$$
a_{\varepsilon}(u, v)=\int_{\Omega} \sigma_{\varepsilon} \nabla u \cdot \nabla v d x, \quad l_{\varepsilon}(v)=\int_{\Omega} f v d x \quad \forall u, v \in H_{0}^{1}(\Omega) .
$$


For the sake of clarity, we will at first concentrate on the inclusion case, and we will afterwards discuss the adaptations to the Neumann case.

For simplicity we consider a cost function of the form

$$
J_{\varepsilon}(u)=\hat{J}\left(u_{\mid \hat{\Omega}}\right),
$$

where $\hat{\Omega}$ is an open subset of $\Omega$ excluding a neighborhood of $z$ and $\hat{J}: H^{1}(\hat{\Omega}) \rightarrow \mathbb{R}$ is Fréchet differentiable. We further assume that $d \hat{J}$ is Lipschitz continuous. Comments will be made regarding more general cost functions in Remark 4.10.

\subsection{Variation of the direct state}

In order to apply Proposition 3.1, it is needed that the variation of the Lagrangian be evaluated at the averaged adjoint state $v_{\varepsilon}$. Since the construction of this adjoint state involves the direct state $u_{\varepsilon}$, our first step is to analyze the behavior of $u_{\varepsilon}$. We adapt the approach of [Gangl and Sturm, 2020].

Set $\tilde{u}_{\varepsilon}=u_{\varepsilon}-u_{0}$. Substracting the variational formulations for $u_{\varepsilon}$ and $u_{0}$ results in

$$
\int_{\Omega} \sigma_{\varepsilon} \nabla \tilde{u}_{\varepsilon} \cdot \nabla \varphi d x=(\alpha-\beta) \int_{\omega_{\varepsilon}} \nabla u_{0} \cdot \nabla \varphi d x \quad \forall \varphi \in H_{0}^{1}(\Omega) .
$$

We now define the rescaled function

$$
U_{\varepsilon}(y)=\frac{1}{\varepsilon} \tilde{u}_{\varepsilon}(z+\varepsilon y), \quad y \in \varepsilon^{-1}(\Omega-z),
$$

so that

$$
\tilde{u}_{\varepsilon}(x)=\varepsilon U_{\varepsilon}\left(\frac{x-z}{\varepsilon}\right), \quad \nabla \tilde{u}_{\varepsilon}(x)=\nabla U_{\varepsilon}\left(\frac{x-z}{\varepsilon}\right) \quad \forall x \in \Omega .
$$

A straightforward change of variables leads to

$$
\int_{\varepsilon^{-1}(\Omega-z)} \sigma_{\varepsilon}(z+\varepsilon y) \nabla U_{\varepsilon}(y) \cdot \nabla \varphi(z+\varepsilon y) d y=(\alpha-\beta) \int_{\omega} \nabla u_{0}(z+\varepsilon y) \cdot \nabla \varphi(z+\varepsilon y) d y \quad \forall \varphi \in H_{0}^{1}(\Omega) .
$$

Changing test functions yields

$$
\int_{\varepsilon^{-1}(\Omega-z)} \sigma_{\varepsilon}(z+\varepsilon y) \nabla U_{\varepsilon}(y) \cdot \nabla \Phi(y) d y=(\alpha-\beta) \int_{\omega} \nabla u_{0}(z+\varepsilon y) \cdot \nabla \Phi(y) d y \quad \forall \Phi \in H_{0}^{1}\left(\varepsilon^{-1}(\Omega-z)\right) .
$$

Let us define the reference conductivity field

$$
\sigma(y)=\left\{\begin{array}{l}
\beta \text { if } y \in \omega \\
\alpha \text { if } y \in \mathbb{R}^{N} \backslash \omega .
\end{array}\right.
$$

With the aim of passing to the limit in (9), we will work with the space

$$
X=\left\{u \in L_{\text {loc }}^{2}\left(\mathbb{R}^{N}\right): \nabla u \in L^{2}\left(\mathbb{R}^{N}\right)\right\},
$$

from the family of Beppo-Levi spaces [Deny and Lions, 1955], and the associated quotient space $X / \mathbb{R}$ by the equivalence relation

$$
u \sim v \Rightarrow \exists c \in \mathbb{R} \text { s.t. } u-v=c .
$$

When it is equipped with the inner product

$$
\langle u, v\rangle_{X / \mathbb{R}}=\int_{\mathbb{R}^{N}} \nabla u \cdot \nabla v d x,
$$


the space $X / \mathbb{R}$ is a Hilbert space [Ortner and Süli, 2012]. By construction the function $U_{\varepsilon}$ belongs to $H_{0}^{1}\left(\varepsilon^{-1}(\Omega-z)\right)$. We implicitly consider an extension by 0 over $\mathbb{R}^{N}$. By the Lax-Milgram theorem there exists a unique $U \in X / \mathbb{R}$ solution of

$$
\int_{\mathbb{R}^{N}} \sigma(y) \nabla U(y) \cdot \nabla \Phi(y) d y=(\alpha-\beta) \int_{\omega} \nabla u_{0}(z) \cdot \nabla \Phi(y) d y \quad \forall \Phi \in X / \mathbb{R} .
$$

The key point is the following convergence result, again adapted from [Gangl and Sturm, 2020]. Alternative estimates can be derived with the help of the fundamental solution of the principal part of the differential operator, see e.g. [Amstutz, 2006a], or by comparison principles [Amstutz and Bonnafé, 2017]. The advantage of the presented approach is that it does not require any knowledge of the behavior of $U$ at infinity. In particular it extends relatively easily to some nonlinear problems [Gangl and Sturm, 2020].

Proposition 4.1 We have the strong convergence $\nabla U_{\varepsilon} \rightarrow \nabla U$ in $L^{2}\left(\mathbb{R}^{N}\right)$ when $\varepsilon \searrow 0$, provided that $\nabla u_{0}$ be continuous at point $z$.

Proof. Step 1. By the extension convention, $H_{0}^{1}\left(\varepsilon^{-1}(\Omega-z)\right) / \mathbb{R}$ is a closed linear subspace of $X / \mathbb{R}$. We denote by $P_{\varepsilon}$ the projection of $U$ onto $H_{0}^{1}\left(\varepsilon^{-1}(\Omega-z)\right) / \mathbb{R}$. By a small abuse of notation we will assume that $P_{\varepsilon}$ stands for the representative in $H_{0}^{1}\left(\varepsilon^{-1}(\Omega-z)\right)$. By definition we have

$$
P_{\varepsilon}=\operatorname{argmin}_{\Phi \in H_{0}^{1}\left(\varepsilon^{-1}(\Omega-z)\right)}\|\nabla \Phi-\nabla U\|_{L^{2}\left(\mathbb{R}^{N}\right)} .
$$

Standard properties of the projection onto a linear subspace ensure that $\left\|\nabla P_{\varepsilon}\right\|_{L^{2}\left(\varepsilon^{-1}(\Omega-z)\right)} \leq\|\nabla U\|_{L^{2}\left(\mathbb{R}^{N}\right)}$ and

$$
\int_{\mathbb{R}^{N}}\left(\nabla P_{\varepsilon}-\nabla U\right) \cdot \nabla \Phi d x=0 \quad \forall \Phi \in H_{0}^{1}\left(\varepsilon^{-1}(\Omega-z)\right) .
$$

The first assertion yields that there exists $Q \in X / \mathbb{R}$ such that $\nabla P_{\varepsilon} \rightarrow \nabla Q$ weakly in $L^{2}\left(\mathbb{R}^{N}\right)$, up to a subsequence. The second assertion implies that

$$
\int_{\mathbb{R}^{N}}(\nabla Q-\nabla U) \cdot \nabla \Phi d x=0 \quad \forall \Phi \in H_{0}^{1}(B(0, R)), \forall R>0
$$

Let $\zeta: \mathbb{R}^{N} \rightarrow[0,1]$ be a smooth function such that $\zeta=1$ in $B(0,1)$ and $\zeta=0$ outside $B(0,2)$. Set $\zeta_{n}(x)=\zeta(x / n)$ and $\Phi_{n}(x)=\left(Q-U+\lambda_{n}\right) \zeta_{n} \in H_{0}^{1}(B(0,2 n))$, with $\lambda_{n} \in \mathbb{R}$ at the moment arbitrary. This yields

$$
\int_{\mathbb{R}^{N}}|\nabla Q-\nabla U|^{2} \zeta_{n} d x+\int_{\mathbb{R}^{N}}\left(Q-U+\lambda_{n}\right)(\nabla Q-\nabla U) \cdot \nabla \zeta_{n} d x=0 .
$$

A change of variables entails

$$
\left\|\left(Q-U+\lambda_{n}\right) \nabla \zeta_{n}\right\|_{L^{2}\left(\mathbb{R}^{N}\right)}^{2}=n^{N-2} \int_{\mathbb{R}^{N}}\left|\left(Q-U+\lambda_{n}\right)(n y) \nabla \zeta(y)\right|^{2} d y \leq c n^{N-2} \int_{R(0,1,2)}\left|\left(Q-U+\lambda_{n}\right)(n y)\right|^{2} d y,
$$

with $R(0,1,2)$ the ring centered at 0 with radii 1 and 2 . We now fix $\lambda_{n}$ such that

$$
\int_{R(0,1,2)}\left(Q-U+\lambda_{n}\right)(n y) d y=0 .
$$

By the Poincaré-Wirtinger inequality we infer

$$
\left\|\left(Q-U+\lambda_{n}\right) \nabla \zeta_{n}\right\|_{L^{2}\left(\mathbb{R}^{N}\right)}^{2} \leq c n^{N} \int_{R(0,1,2)}|\nabla(Q-U)(n y)|^{2} d y \leq c \int_{R(0, n, 2 n)}|\nabla(Q-U)|^{2} d x .
$$


Plugging this into (11) and using the Cauchy-Schwarz inequality, we arrive at

$$
\int_{\mathbb{R}^{N}}|\nabla Q-\nabla U|^{2} \zeta_{n} d x \leq c\|\nabla Q-\nabla U\|_{L^{2}\left(\mathbb{R}^{N}\right)}\left(\int_{R(0, n, 2 n)}|\nabla Q-\nabla U|^{2} d x\right)^{1 / 2} .
$$

Letting now $n$ go to $+\infty$ results in $\nabla Q=\nabla U$. Therefore the whole sequence $\nabla P_{\varepsilon}$ weakly converges to $\nabla U$. In particular we infer that

$$
\int_{\mathbb{R}^{N}} \nabla P_{\varepsilon} \cdot \nabla U d x \rightarrow \int_{\mathbb{R}^{N}}|\nabla U|^{2} d x
$$

From the identity

$$
\left\|\nabla P_{\varepsilon}-\nabla U\right\|_{L^{2}\left(\mathbb{R}^{N}\right)}^{2}=\left\|\nabla P_{\varepsilon}\right\|_{L^{2}\left(\mathbb{R}^{N}\right)}^{2}+\|\nabla U\|_{L^{2}\left(\mathbb{R}^{N}\right)}^{2}-2 \int_{\mathbb{R}^{N}} \nabla P_{\varepsilon} \cdot \nabla U d x
$$

we derive

$$
\limsup _{\varepsilon \rightarrow 0}\left\|\nabla P_{\varepsilon}-\nabla U\right\|_{L^{2}\left(\mathbb{R}^{N}\right)}^{2} \leq \limsup _{\varepsilon \rightarrow 0}\left\|\nabla P_{\varepsilon}\right\|_{L^{2}\left(\mathbb{R}^{N}\right)}^{2}-\|\nabla U\|_{L^{2}\left(\mathbb{R}^{N}\right)}^{2} \leq 0 .
$$

We have shown that $\nabla P_{\varepsilon}$ strongly converges to $\nabla U$ in $L^{2}\left(\mathbb{R}^{N}\right)$.

Step 2. Using (9) we obtain

$$
\begin{aligned}
& \int_{\varepsilon^{-1}(\Omega-z)} \sigma(y) \nabla\left(P_{\varepsilon}-U_{\varepsilon}\right)(y) \cdot \nabla \Phi(y) d y=\int_{\varepsilon^{-1}(\Omega-z)} \sigma(y) \nabla P_{\varepsilon}(y) \cdot \nabla \Phi(y) d y \\
&-(\alpha-\beta) \int_{\omega} \nabla u_{0}(z+\varepsilon y) \cdot \nabla \Phi(y) d y \quad \forall \Phi \in H_{0}^{1}\left(\varepsilon^{-1}(\Omega-z)\right) .
\end{aligned}
$$

In view of (10) this rewrites as

$$
\begin{aligned}
\int_{\varepsilon^{-1}(\Omega-z)} \sigma(y) \nabla\left(P_{\varepsilon}-U_{\varepsilon}\right)(y) \cdot \nabla \Phi(y) d y=\int_{\varepsilon^{-1}(\Omega-z)} \sigma(y) \nabla\left(P_{\varepsilon}-U\right)(y) \cdot \nabla \Phi(y) d y \\
-(\alpha-\beta) \int_{\omega}\left(\nabla u_{0}(z+\varepsilon y)-\nabla u_{0}(z)\right) \cdot \nabla \Phi(y) d y \quad \forall \Phi \in H_{0}^{1}\left(\varepsilon^{-1}(\Omega-z)\right) .
\end{aligned}
$$

Choose $\Phi=P_{\varepsilon}-U_{\varepsilon}$. We obtain for some constant $c$

$$
\begin{aligned}
\left\|\nabla\left(P_{\varepsilon}-U_{\varepsilon}\right)\right\|_{L^{2}\left(\varepsilon^{-1}(\Omega-z)\right)}^{2} \leq c\left(\left\|\nabla\left(P_{\varepsilon}-U\right)\right\|_{L^{2}\left(\varepsilon^{-1}(\Omega-z)\right)}\left\|\nabla\left(P_{\varepsilon}-U_{\varepsilon}\right)\right\|_{L^{2}\left(\varepsilon^{-1}(\Omega-z)\right)}\right. \\
\left.+\left\|\nabla u_{0}(z+\varepsilon y)-\nabla u_{0}(z)\right\|_{L^{2}(\omega)}\left\|\nabla\left(P_{\varepsilon}-U_{\varepsilon}\right)\right\|_{L^{2}\left(\varepsilon^{-1}(\Omega-z)\right)}\right),
\end{aligned}
$$

leading to

$$
\left\|\nabla\left(P_{\varepsilon}-U_{\varepsilon}\right)\right\|_{L^{2}\left(\varepsilon^{-1}(\Omega-z)\right)} \leq c\left(\left\|\nabla\left(P_{\varepsilon}-U\right)\right\|_{L^{2}\left(\varepsilon^{-1}(\Omega-z)\right)}+\left\|\nabla u_{0}(z+\varepsilon y)-\nabla u_{0}(z)\right\|_{L^{2}(\omega)}\right) .
$$

Using step 1 and the continuity assumption we arrive at $\left\|\nabla\left(P_{\varepsilon}-U_{\varepsilon}\right)\right\|_{L^{2}\left(\varepsilon^{-1}(\Omega-z)\right)} \rightarrow 0$.

Conclusion. The proof is completed by combining step 1 and step 2 .

Corollary 4.2 Under the assumption of Proposition 4.1 we have

$$
\left\|\tilde{u}_{\varepsilon}\right\|_{H^{1}(\Omega)}^{2}=O\left(\varepsilon^{N}\right),
$$

and for any $R>0$

$$
\left\|\tilde{u}_{\varepsilon}\right\|_{H^{1}(\Omega \backslash B(z, R))}^{2}=o\left(\varepsilon^{N}\right)
$$


PROOF. By change of variables it is straightforward that

$$
\left\|\nabla \tilde{u}_{\varepsilon}\right\|_{L^{2}(\Omega)}^{2}=\varepsilon^{N}\left\|\nabla U_{\varepsilon}\right\|_{L^{2}\left(\varepsilon^{-1}(\Omega-z)\right)}^{2} .
$$

Proposition 4.1 yields that $\left\|\nabla U_{\varepsilon}\right\|_{L^{2}\left(\varepsilon^{-1}(\Omega-z)\right)}=O(1)$, whereby $\left\|\tilde{u}_{\varepsilon}\right\|_{H^{1}(\Omega)}^{2}=O\left(\varepsilon^{N}\right)$ by the Poincaré inequality. Let now $R>0$. The same change of variables provides

$$
\left\|\nabla \tilde{u}_{\varepsilon}\right\|_{L^{2}(\Omega \backslash B(z, R))}^{2}=\varepsilon^{N}\left\|\nabla U_{\varepsilon}\right\|_{L^{2}\left(\varepsilon^{-1}(\Omega-z)\right) \backslash B\left(0, \varepsilon^{-1} R\right)}^{2}=\varepsilon^{N}\left\|\nabla U_{\varepsilon}\right\|_{L^{2}\left(\mathbb{R}^{N} \backslash B\left(0, \varepsilon^{-1} R\right)\right.}^{2} .
$$

This can be rephrased as

$$
\left\|\nabla \tilde{u}_{\varepsilon}\right\|_{L^{2}(\Omega \backslash B(z, R))}^{2}=\varepsilon^{N} \int_{\mathbb{R}^{N}}\left(1-\chi_{B\left(0, \frac{R}{\varepsilon}\right)}(y)\right)\left|\nabla U_{\varepsilon}(y)\right|^{2} d y .
$$

Using Proposition 4.1 we infer $\left\|\nabla \tilde{u}_{\varepsilon}\right\|_{L^{2}(\Omega \backslash B(z, R))}^{2}=o\left(\varepsilon^{N}\right)$. The claim is achieved by the Poincaré inequality.

\subsection{Variation of the adjoint state}

In view of Proposition 3.1 we define the adjoint state $v_{\varepsilon} \in H_{0}^{1}(\Omega)$ solution of

$$
\int_{\Omega} \sigma_{\varepsilon} \nabla v_{\varepsilon} \cdot \nabla \varphi d x=-\int_{0}^{1} d J_{\varepsilon}\left(t u_{\varepsilon}+(1-t) u_{0}\right) \varphi d t \quad \forall \varphi \in H_{0}^{1}(\Omega) .
$$

In particular the unperturbed adjoint state $v_{0}$ satisfies

$$
\int_{\Omega} \alpha \nabla v_{0} \cdot \nabla \varphi d x=-d J_{0}\left(u_{0}\right) \varphi \quad \forall \varphi \in H_{0}^{1}(\Omega)
$$

As for the direct state we set $\tilde{v}_{\varepsilon}=v_{\varepsilon}-v_{0}$. We obtain by difference the equality, valid for all $\varphi \in H_{0}^{1}(\Omega)$,

$$
\int_{\Omega} \sigma_{\varepsilon} \nabla \tilde{v}_{\varepsilon} \cdot \nabla \varphi d x=(\alpha-\beta) \int_{\omega_{\varepsilon}} \nabla v_{0} \cdot \nabla \varphi d x-\int_{0}^{1} d J_{\varepsilon}\left(t u_{\varepsilon}+(1-t) u_{0}\right) \varphi d t+d J_{0}\left(u_{0}\right) \varphi .
$$

By (8) this rewrites

$$
\int_{\Omega} \sigma_{\varepsilon} \nabla \tilde{v}_{\varepsilon} \cdot \nabla \varphi d x=(\alpha-\beta) \int_{\omega_{\varepsilon}} \nabla v_{0} \cdot \nabla \varphi d x-\int_{0}^{1}\left(d \hat{J}\left(\left(t u_{\varepsilon}+(1-t) u_{0}\right)_{\mid \hat{\Omega}}\right)-d \hat{J}\left(u_{0 \mid \hat{\Omega}}\right)\right) \varphi_{\mid \hat{\Omega}} d t .
$$

We will later justify that the latter integral can be disregarded, therefore we define $w_{\varepsilon} \in H_{0}^{1}(\Omega)$ solution of

$$
\int_{\Omega} \sigma_{\varepsilon} \nabla w_{\varepsilon} \cdot \nabla \varphi d x=(\alpha-\beta) \int_{\omega_{\varepsilon}} \nabla v_{0} \cdot \nabla \varphi d x \quad \forall \varphi \in H_{0}^{1}(\Omega) .
$$

In order to approximate this $w_{\varepsilon}$ we proceed exactly as for the direct state. We define

$$
W_{\varepsilon}(y)=\frac{1}{\varepsilon} w_{\varepsilon}(z+\varepsilon y), \quad y \in \varepsilon^{-1}(\Omega-z),
$$

and $W \in X / \mathbb{R}$ solution of

$$
\int_{\mathbb{R}^{N}} \sigma(y) \nabla W(y) \cdot \nabla \Phi(y) d y=(\alpha-\beta) \int_{\omega} \nabla v_{0}(z) \cdot \nabla \Phi(y) d y \quad \forall \Phi \in X / \mathbb{R} .
$$

We obtain: 
Proposition 4.3 It holds $\nabla W_{\varepsilon} \rightarrow \nabla W$ in $L^{2}\left(\mathbb{R}^{N}\right)$ when $\varepsilon \searrow 0$, provided that $\nabla v_{0}$ be continuous at point $z$.

We now analyze the approximation of $\tilde{v}_{\varepsilon}$ by $w_{\varepsilon}$.

Lemma 4.4 It holds

$$
\left\|\tilde{v}_{\varepsilon}-w_{\varepsilon}\right\|_{H^{1}(\Omega)}^{2}=o\left(\varepsilon^{N}\right) .
$$

PROOF. Set $e_{\varepsilon}=\tilde{v}_{\varepsilon}-w_{\varepsilon}$. It solves

$$
\int_{\Omega} \sigma_{\varepsilon} \nabla e_{\varepsilon} \cdot \nabla \varphi d x=-\int_{0}^{1}\left(d \hat{J}\left(\left(t u_{\varepsilon}+(1-t) u_{0}\right)_{\mid \hat{\Omega}}\right)-d \hat{J}\left(u_{0 \mid \hat{\Omega}}\right)\right) \varphi_{\mid \hat{\Omega}} d t \quad \forall \varphi \in H_{0}^{1}(\Omega) .
$$

We choose $\varphi=e_{\varepsilon}$. Using that $d \hat{J}$ is Lipschitz we obtain

$$
\left\|\nabla e_{\varepsilon}\right\|_{L^{2}(\Omega)}^{2} \leq c \int_{0}^{1} t\left\|u_{\varepsilon}-u_{0}\right\|_{H^{1}(\hat{\Omega})}\left\|e_{\varepsilon}\right\|_{H^{1}(\hat{\Omega})} d t .
$$

Corollary 4.2 and the Poincaré inequality provide the desired estimate.

\subsection{Variation of the Lagrangian}

Following Proposition 3.1 and (7) we define the Lagrangian

$$
\mathcal{L}_{\varepsilon}(u, v)=J_{\varepsilon}(u)+\int_{\Omega} \sigma_{\varepsilon} \nabla u \cdot \nabla v d x-\int_{\Omega} f v d x . \quad \forall u, v \in H_{0}^{1}(\Omega) .
$$

We need to estimate the variation

$$
\left(\mathcal{L}_{\varepsilon}-\mathcal{L}_{0}\right)\left(u_{0}, v_{\varepsilon}\right)=(\beta-\alpha) \int_{\omega_{\varepsilon}} \nabla u_{0} \cdot \nabla v_{\varepsilon} d x
$$

which can be decomposed as

$\left(\mathcal{L}_{\varepsilon}-\mathcal{L}_{0}\right)\left(u_{0}, v_{\varepsilon}\right)=(\beta-\alpha) \int_{\omega_{\varepsilon}} \nabla u_{0} \cdot \nabla v_{0} d x+(\beta-\alpha) \int_{\omega_{\varepsilon}} \nabla u_{0} \cdot \nabla w_{\varepsilon} d x+(\beta-\alpha) \int_{\omega_{\varepsilon}} \nabla u_{0} \cdot\left(\nabla \tilde{v}_{\varepsilon}-\nabla w_{\varepsilon}\right) d x$

Lemma 4.5 If $\nabla u_{0}$ and $\nabla v_{0}$ are continuous at point $z$ then

$$
\left(\mathcal{L}_{\varepsilon}-\mathcal{L}_{0}\right)\left(u_{0}, v_{\varepsilon}\right)=\varepsilon^{N}(\beta-\alpha)|\omega| \nabla u_{0}(z) \cdot \nabla v_{0}(z)+(\beta-\alpha) \int_{\omega_{\varepsilon}} \nabla u_{0} \cdot \nabla w_{\varepsilon} d x+o\left(\varepsilon^{N}\right) .
$$

Proof. We first estimate

$$
\begin{aligned}
\int_{\omega_{\varepsilon}} \nabla u_{0} \cdot \nabla v_{0} d x-\varepsilon^{N}|\omega| \nabla u_{0}(z) \cdot \nabla v_{0}(z) & =\int_{\omega_{\varepsilon}}\left(\nabla u_{0} \cdot \nabla v_{0}-\nabla u_{0}(z) \cdot \nabla v_{0}(z)\right) d x \\
& =\varepsilon^{N} \int_{\omega}\left(\nabla u_{0}(z+\varepsilon y) \cdot \nabla v_{0}(z+\varepsilon y)-\nabla u_{0}(z) \cdot \nabla v_{0}(z)\right) d y \\
& =o\left(\varepsilon^{N}\right) .
\end{aligned}
$$

Secondly, the Cauchy-Schwarz inequality yields

$$
\left|\int_{\omega_{\varepsilon}} \nabla u_{0} \cdot\left(\nabla \tilde{v}_{\varepsilon}-\nabla w_{\varepsilon}\right) d x\right| \leq\left\|\nabla u_{0}\right\|_{L^{2}\left(\omega_{\varepsilon}\right)}\left\|\nabla \tilde{v}_{\varepsilon}-\nabla w_{\varepsilon}\right\|_{L^{2}\left(\omega_{\varepsilon}\right)}=O\left(\varepsilon^{N / 2}\right) o\left(\varepsilon^{N / 2}\right),
$$

by Lemma 4.4 .

From the expression found in Lemma 4.5 we make a change of variables to obtain

$$
\left(\mathcal{L}_{\varepsilon}-\mathcal{L}_{0}\right)\left(u_{0}, v_{\varepsilon}\right)=\varepsilon^{N}(\beta-\alpha)|\omega| \nabla u_{0}(z) \cdot \nabla v_{0}(z)+\varepsilon^{N}(\beta-\alpha) \int_{\omega} \nabla u_{0}(z+\varepsilon y) \cdot \nabla W_{\varepsilon}(y) d y+o\left(\varepsilon^{N}\right) .
$$


Lemma 4.6 If $\nabla u_{0}$ and $\nabla v_{0}$ are continuous at point $z$ then

$$
\left(\mathcal{L}_{\varepsilon}-\mathcal{L}_{0}\right)\left(u_{0}, v_{\varepsilon}\right)=\varepsilon^{N}(\beta-\alpha)|\omega| \nabla u_{0}(z) \cdot \nabla v_{0}(z)+\varepsilon^{N}(\beta-\alpha) \int_{\omega} \nabla u_{0}(z) \cdot \nabla W(y) d y+o\left(\varepsilon^{N}\right) .
$$

Proof. We have to show that

$$
\lim _{\varepsilon \rightarrow 0} \int_{\omega}\left(\nabla u_{0}(z+\varepsilon y) \cdot \nabla W_{\varepsilon}(y)-\nabla u_{0}(z) \cdot \nabla W(y)\right) d y=0 .
$$

It is an immediate consequence of Proposition 4.3, using

$$
\begin{aligned}
\int_{\omega}\left(\nabla u_{0}(z+\varepsilon y) \cdot\right. & \left.\nabla W_{\varepsilon}(y)-\nabla u_{0}(z) \cdot \nabla W(y)\right) d y \\
& =\int_{\omega}\left(\nabla u_{0}(z+\varepsilon y)-\nabla u_{0}(z)\right) \cdot \nabla W_{\varepsilon}(y) d y+\int_{\omega} \nabla u_{0}(z) \cdot\left(\nabla W_{\varepsilon}(y)-\nabla W(y)\right) d y
\end{aligned}
$$

and the Cauchy-Schwarz inequality.

\subsection{Polarization matrix}

The definition (13) of $W$ shows that $W$ depends linearly on $\nabla v_{0}(z)$. Denoting by $\left(e_{1}, \cdots, e_{N}\right)$ the canonical basis of $\mathbb{R}^{N}$ and by $\zeta_{i} \in X / \mathbb{R}$ the solution of

$$
\int_{\mathbb{R}^{N}} \sigma(y) \nabla \zeta_{i}(y) \cdot \nabla \Phi(y) d y=(\alpha-\beta) \int_{\omega} e_{i} \cdot \nabla \Phi(y) d y \quad \forall \Phi \in X / \mathbb{R}
$$

we identify that $W(y)=\nabla v_{0}(z) \cdot \zeta(y)$. It follows that

$$
\int_{\omega} \nabla u_{0}(z) \cdot \nabla W(y) d y=\nabla u_{0}(z) \cdot\left(\int_{\omega} D \zeta(y)^{\top} d y\right) \nabla v_{0}(z)
$$

Choosing $\Phi=\zeta_{j}$ in (14) yields

$$
(\alpha-\beta) \int_{\omega} e_{i} \cdot \nabla \zeta_{j}(y) d y=\int_{\mathbb{R}^{N}} \sigma(y) \nabla \zeta_{i}(y) \cdot \nabla \zeta_{j}(y) d y=(\alpha-\beta) \int_{\omega} e_{j} \cdot \nabla \zeta_{i}(y) d y .
$$

This shows that

$$
Q:=\int_{\omega} D \zeta(y) d y=Q^{\top}
$$

We arrive at

$$
|\omega| \nabla u_{0}(z) \cdot \nabla v_{0}(z)+\int_{\omega} \nabla u_{0}(z) \cdot \nabla W(y) d y=\nabla u_{0}(z) \cdot(|\omega| I+Q) \nabla v_{0}(z)
$$

Definition 4.7 We call polarization matrix the symmetric matrix

$$
\mathcal{P}=\left(\frac{\beta}{\alpha}-1\right)(|\omega| I+Q)
$$

Note that (14) can be equivalently rewritten as

$$
\int_{\mathbb{R}^{N}} \sigma(y) \nabla \zeta_{i}(y) \cdot \nabla \Phi(y) d y=(\alpha-\beta) \int_{\partial \omega} e_{i} \cdot n(y) \Phi(y) d y \quad \forall \Phi \in X / \mathbb{R},
$$

therefore the corresponding strong form reads

$$
\left\{\begin{array}{l}
\Delta \zeta_{i}=0 \text { in } \mathbb{R}^{N} \backslash \partial \omega \\
\beta\left(\frac{\partial \zeta_{i}}{\partial n}\right)_{\text {int }}-\alpha\left(\frac{\partial \zeta_{i}}{\partial n}\right)_{\text {ext }}=(\alpha-\beta) e_{i} \cdot n \text { on } \partial \omega
\end{array}\right.
$$

Let us now give an additional property of the polarization matrix. 
Proposition 4.8 The eigenvalues $\left(\lambda_{i}\right)$ of the polarization matrix satisfy the inequality

$$
\lambda_{i} \leq\left(\frac{\beta}{\alpha}-1\right)|\omega|
$$

Moreover the polarization matrix is

- symmetric positive definite if $\beta>\alpha$,

- symmetric negative definite if $\beta<\alpha$.

Proof. Since $\mathcal{P}$ is symmetric, let us choose an othogonal basis in which it is diagonal.

Choosing $\Phi=\zeta_{i}$ in (14) entails

$$
(\alpha-\beta) \int_{\omega} e_{i} \cdot \nabla \zeta_{i}(y) d y \geq 0
$$

We infer that

$$
\mathcal{P} e_{i} \cdot e_{i}=\left(\frac{\beta}{\alpha}-1\right)\left(|\omega|+\int_{\omega} e_{i} \cdot \nabla \zeta_{i}(y) d y\right) \leq\left(\frac{\beta}{\alpha}-1\right)|\omega| .
$$

Case $\beta<\alpha$. The above inequality directly shows that $\mathcal{P} e_{i} \cdot e_{i}<0$, hence $\mathcal{P}$ is symmetric negative definite.

Case $\beta>\alpha$. We write

$$
\mathcal{P} e_{i} \cdot e_{i}=\left(\frac{\beta}{\alpha}-1\right) \int_{\omega}\left(\nabla \zeta_{i}+e_{i}\right) \cdot e_{i} d y=\left(\frac{\beta}{\alpha}-1\right) \int_{\omega}\left(\left|\nabla \zeta_{i}+e_{i}\right|^{2}-\left(\nabla \zeta_{i}+e_{i}\right) \cdot \nabla \zeta_{i}\right) d y
$$

Using (14) we obtain

$$
(\alpha-\beta) \int_{\omega}\left(\nabla \zeta_{i}+e_{i}\right) \cdot \nabla \zeta_{i} d y=(\alpha-\beta) \int_{\omega}\left|\nabla \zeta_{i}\right|^{2} d y+\int_{\mathbb{R}^{N}} \sigma\left|\nabla \zeta_{i}\right|^{2} d y=\alpha \int_{\mathbb{R}^{N}}\left|\nabla \zeta_{i}\right|^{2} d y .
$$

This yields

$$
\mathcal{P} e_{i} \cdot e_{i}=\left(\frac{\beta}{\alpha}-1\right) \int_{\omega}\left|\nabla \zeta_{i}+e_{i}\right|^{2} d y+\int_{\mathbb{R}^{N}}\left|\nabla \zeta_{i}\right|^{2} d y>0,
$$

hence $\mathcal{P}$ is symmetric positive definite.

The notion of polarization matrix goes back at least to [Schiffer and Szegö, 1949, Pólya and Szegö, 1951]. Detailed properties and generalizations can be found in [Ammari and Kang, 2007]. In case of a vectorvalued state, a polarization tensor is involved. In the framework of linear elasticity it is called the elastic moments tensor.

We end this section by providing closed forms of the polarization matrix for ball-shaped inclusions, i.e., when $\omega$ is the unit ball $B(0,1)$. The main step is to solve (17). The typical method is to "guess" a candidate solution, including free parameters, and to plug this ansatz in the system to fix the parameters. For instance, in the 2D case, we find the solution

$$
\zeta_{i}(y)=\frac{\alpha-\beta}{\alpha+\beta} \times\left\{\begin{array}{l}
e_{i} \cdot y \text { in } \omega \\
\frac{e_{i} \cdot y}{|y|^{2}} \text { in } \mathbb{R}^{2} \backslash \bar{\omega}
\end{array}\right.
$$

Results are gathered in Table 2. 


\begin{tabular}{|c|c|c|}
\hline $1 \mathrm{D}$ & $2 \mathrm{D}$ & $3 \mathrm{D}$ \\
\hline $\mathcal{P}=2\left(1-\frac{\alpha}{\beta}\right)$ & $\mathcal{P}=2 \pi \frac{\beta-\alpha}{\beta+\alpha} I$ & $\mathcal{P}=4 \pi \frac{\beta-\alpha}{\beta+2 \alpha} I$ \\
\hline
\end{tabular}

Table 2: Polarization matrix in the inclusion case for $\omega=B(0,1)$

\subsection{Expression of the topological asymptotic expansion}

Let us recapitulate our findings.

Theorem 4.9 Consider a cost function of form (8). Let $v_{0} \in H_{0}^{1}(\Omega)$ be the solution of (12). Then

$$
J_{\varepsilon}\left(u_{\varepsilon}\right)-J_{0}\left(u_{0}\right)=\varepsilon^{N} \alpha \nabla u_{0}(z) \cdot \mathcal{P} \nabla v_{0}(z)+o\left(\varepsilon^{N}\right),
$$

where $\mathcal{P}$ is the polarization matrix.

Proof. It follows from Proposition 3.1, Lemma 4.6, (15) and Definition 4.7. The regularity conditions for $u_{0}$ and $v_{0}$ are ensured by elliptic regularity.

As a consequence of Theorem 4.9 and Definition 1.1 we can set the topological derivative of a shape functional $\mathcal{J}$ such that $\mathcal{J}\left(\Omega_{\varepsilon}\right)=J_{\varepsilon}\left(u_{\varepsilon}\right)$ as

$$
d_{T} \mathcal{J}(\Omega, \omega, z)=\alpha \nabla u_{0}(z) \cdot \mathcal{P} \nabla v_{0}(z) .
$$

Remark 4.10 Other types of cost functions may yield additional terms in the topological derivative, see e.g. [Amstutz, 2006a, Amstutz et al., 2014, Amstutz et al., 2012, Gangl and Sturm, 2020, Novotny and Sokolowski, 2013].

\subsection{The Neumann case}

The Neumann case $(\beta=0)$ can be analyzed along the same lines as the inclusion case, with some minor modifications. In particular, some volume integrals over the inclusion have to be replaced by boundary integrals using the Green formula. The function $U$ is now defined in the space $X_{\omega} / \mathbb{R}$, with

$$
X_{\omega}=\left\{u \in L_{\mathrm{loc}}^{2}\left(\mathbb{R}^{N} \backslash \omega\right): \nabla u \in L^{2}\left(\mathbb{R}^{N} \backslash \bar{\omega}\right)\right\},
$$

by, instead of (10),

$$
\int_{\mathbb{R}^{N} \backslash \omega} \nabla U(y) \cdot \nabla \Phi(y) d y=\int_{\partial \omega} \nabla u_{0}(z) \cdot n \Phi d s \quad \forall \Phi \in X_{\omega} / \mathbb{R} .
$$

In order to use the semi-norm as a norm on the quotient space $X_{\omega} / \mathbb{R}$, it is required that $\mathbb{R}^{N} \backslash \omega$ be connected. This is in principle a rather mild assumption, except in dimension 1 where it cannot hold. Actually, it is clear that in dimension 1 the cost function is likely to be discontinuous at $\varepsilon=0$. This singularity can also be seen by observing that the polarization of the inclusion diverges when $\beta \rightarrow 0$, see Table 2. The same phenomenon occurs in higher dimension with higher order differential operators [Amstutz et al., 2014].

Eventually, in dimension $N \geq 2$ with $\mathbb{R}^{N} \backslash \omega$ connected, we arrive at the same result as in Theorem 4.9 with the polarization matrix defined through $\zeta_{i} \in X_{\omega} / \mathbb{R}$,

$$
\int_{\mathbb{R}^{N} \backslash \omega} \nabla \zeta_{i}(y) \cdot \nabla \Phi(y) d y=\int_{\partial \omega} e_{i} \cdot n \Phi(y) d y \quad \forall \Phi \in X_{\omega} / \mathbb{R}, \quad Q:=\int_{\partial \omega} \zeta \otimes n d s .
$$




\begin{tabular}{|c|c|c|}
\hline ball $(2 \mathrm{D}, 3 \mathrm{D})$ & straight crack (2D) & circular crack (3D) \\
\hline $\mathcal{P}=-2 \pi I$ & $\mathcal{P}=-\pi \nu \otimes \nu$ & $\mathcal{P}=-\frac{8}{3} \nu \otimes \nu$ \\
\hline
\end{tabular}

Table 3: Polarization matrix in the Neumann case

Note that (17) still holds, with $\beta=0$. Therefore, the expressions given in Table 2 apply with $\beta=0$.

An interesting extension is the case of a crack with Neumann boundary condition on each face. A particular case is the planar crack $\omega=\left\{x \in \mathbb{R}^{N}: x \cdot \nu=0,|x|<1\right\}$ of unit normal $\nu$. The corresponding polarization matrix in dimensions 2 and 3 are reported in Table 3, see [Amstutz et al., 2005, Amstutz and Dominguez, 2008, Bonnet, 2011, Bellis and Bonnet, 2013].

\subsection{Extensions}

The topological derivative concept in the inclusion or Neumann cases has been developed in many directions. To give a non-exhaustive overview, let us mention the linear elasticity case [Garreau et al., 2001, Bonnet and Delgado, 2013], the Maxwell equations [Masmoudi et al., 2005], Stokes flows [Ben Abda et al., 0910], nonlinear problems [Amstutz and Bonnafé, 2017, Gangl and Sturm, 2020], evolution problems [Bonnet, 2006, Amstutz et al., 2008, Bellis and Bonnet, 2013], higher order differential equations [Amstutz et al., 2014, Aubert and Drogoul, 2015], higher order topological derivatives [Bonnet, 2009, Bonnet, 2011, Hintermüller et al., 2012, Bonnet and Cornaggia, 2017, Novotny et al., 2019b]; see also the monograph [Novotny and Sokolowski, 2013].

\section{Dirichlet case in $2 \mathrm{D}$}

\subsection{Problem formulation}

Let $\Omega$ be an open and bounded subset of $\mathbb{R}^{2}$ and $\omega$ be a bounded, smooth open subset of $\mathbb{R}^{2}$. Like in the previous section we consider a point $z \in \Omega$ and, for $\varepsilon \geq 0$ small enough, the perforated domain

$$
\Omega_{\varepsilon}=\Omega \backslash \overline{\omega_{\varepsilon}}, \quad \omega_{\varepsilon}=z+\varepsilon \omega \subset \Omega .
$$

We assume for convenience, but without loss of generality, that $0 \in \omega \subset \subset B(0,1)$. We address the problem

$$
\left\{\begin{array}{l}
-\Delta u_{\varepsilon}=f \text { in } \Omega_{\varepsilon} \\
u_{\varepsilon}=0 \text { on } \partial \Omega_{\varepsilon}
\end{array}\right.
$$

It is assumed that $f \in L^{2}(\Omega)$ with $f=0$ in a neighborhood of $z$. We will implicitly suppose that $\varepsilon$ is small enough so that $f=0$ in $\omega_{\varepsilon}$. We denote by $u_{0}$ the unperturbed state, solution of

$$
\left\{\begin{array}{l}
-\Delta u_{0}=f \text { in } \Omega \\
u_{0}=0 \text { on } \partial \Omega
\end{array}\right.
$$

In order to develop the adjoint method of section 3 in a fixed space we extend $u_{\varepsilon}$ by 0 inside $\omega_{\varepsilon}$. We set

$$
\begin{gathered}
a_{\varepsilon}(u, v)=\int_{\Omega} \nabla u \cdot \nabla v d x \quad \forall u, v \in H_{0}^{1}(\Omega) \\
l_{\varepsilon}(v)=\int_{\Omega} \nabla u_{\varepsilon} \cdot \nabla v d x=\int_{\Omega_{\varepsilon}} \nabla u_{\varepsilon} \cdot \nabla v d x=\int_{\Omega_{\varepsilon}} f v d x-\int_{\partial \omega_{\varepsilon}} \frac{\partial u_{\varepsilon}}{\partial n} v d s \quad \forall v \in H_{0}^{1}(\Omega) .
\end{gathered}
$$


By convention the normal to $\partial \omega_{\varepsilon}$ is chosen outward to $\omega_{\varepsilon}$. This construction ensures that $u_{\varepsilon} \in H_{0}^{1}(\Omega)$ satisfies

$$
a_{\varepsilon}\left(u_{\varepsilon}, v\right)=l_{\varepsilon}(v) \quad \forall v \in H_{0}^{1}(\Omega) .
$$

We again consider a cost function of the form

$$
J_{\varepsilon}(u)=\hat{J}\left(u_{\mid \hat{\Omega}}\right),
$$

where $\hat{\Omega}$ is an open subset of $\Omega$ excluding a neighborhood of $z$ and $\hat{J}: H^{1}(\hat{\Omega}) \rightarrow \mathbb{R}$ is Fréchet differentiable. We further assume that $d \hat{J}$ is Lipschitz continuous.

\subsection{A preliminary estimate}

We shall work with the weighted Sobolev space [Dautray and Lions, 1988, Amrouche et al., 1994]

$$
W\left(\mathbb{R}^{2}\right)=\left\{u \in L_{\mathrm{loc}}^{2}\left(\mathbb{R}^{2}\right): w u \in L^{2}\left(\mathbb{R}^{2}\right), \nabla u \in L^{2}\left(\mathbb{R}^{2}\right)\right\},
$$

with the weight function

$$
w(x)=\frac{1}{\sqrt{1+|x|^{2}} \log (2+|x|)} .
$$

It is a Hilbert space for the inner product

$$
\langle u, v\rangle_{W\left(\mathbb{R}^{2}\right)}=\int_{\mathbb{R}^{2}}\left(w^{2} u v+\nabla u \cdot \nabla v\right) d x .
$$

We also define the subspace

$$
W_{0}\left(\mathbb{R}^{2} \backslash \bar{\omega}\right)=\left\{u \in W\left(\mathbb{R}^{2} \backslash \bar{\omega}\right): u=0 \text { on } \partial \omega\right\} .
$$

We have the following standard Poincaré inequality, for which we sketch a proof for completeness.

Proposition 5.1 There exists $c>0$ such that

$$
\|u\|_{W\left(\mathbb{R}^{2} \backslash \bar{\omega}\right)} \leq c\|\nabla u\|_{L^{2}\left(\mathbb{R}^{2} \backslash \bar{\omega}\right)} \quad \forall u \in W_{0}\left(\mathbb{R}^{2} \backslash \bar{\omega}\right) .
$$

Proof. Step 1. Consider first a function $u \in \mathcal{C}_{c}^{\infty}\left(\mathbb{R}^{2} \backslash \bar{B}(0, a)\right), a>1$. For an arbitrary unit vector $e$ we set $f(r)=u(r e)$. Integration by parts yields

$$
\int_{a}^{+\infty} \frac{1}{r \log ^{2} r} f(r)^{2} d r=\int_{a}^{+\infty} \frac{2}{\log r} f(r) f^{\prime}(r) d r
$$

whereby we obtain by the Cauchy-Schwarz inequality

$$
\int_{a}^{+\infty} \frac{1}{r \log ^{2} r} f(r)^{2} d r \leq 2\left(\int_{a}^{+\infty} \frac{1}{r \log ^{2} r} f(r)^{2} d r\right)^{1 / 2}\left(\int_{a}^{+\infty} r f^{\prime}(r)^{2} d r\right)^{1 / 2} .
$$

This implies

$$
\int_{a}^{+\infty} \frac{1}{r \log ^{2} r} f(r)^{2} d r \leq 4 \int_{a}^{+\infty} r f^{\prime}(r)^{2} d r
$$

thence

$$
\|u\|_{W\left(\mathbb{R}^{2} \backslash \bar{B}(0, a)\right)} \leq \sqrt{5}\|\nabla u\|_{L^{2}\left(\mathbb{R}^{2} \backslash \bar{B}(0, a)\right)} .
$$

By a density argument, this holds for all $u \in W_{0}\left(\mathbb{R}^{2} \backslash \bar{B}(0, a)\right)$. 
Step 2. Let now $u \in W_{0}\left(\mathbb{R}^{2} \backslash \bar{\omega}\right)$, and $a>1$. Let $\theta \in \mathcal{C}_{c}^{\infty}\left(\mathbb{R}^{2}\right)$ such that $\theta=1$ in $B(0,2 a)$ and $\theta=0$ outside $B(0,3 a)$. By step 1 we have

$$
\|(1-\theta) u\|_{W\left(\mathbb{R}^{2} \backslash \bar{\omega}\right)} \leq c\|(1-\theta) \nabla u-u \nabla \theta\|_{L^{2}\left(\mathbb{R}^{2} \backslash \bar{\omega}\right)} \leq c\left(\|\nabla u\|_{L^{2}\left(\mathbb{R}^{2} \backslash \bar{\omega}\right)}+\|u\|_{L^{2}(B(0,3 a) \backslash \bar{\omega})}\right) .
$$

The Poincaré inequality in $\left\{v \in H^{1}(B(0,3 a) \backslash \bar{\omega})\right): v=0$ on $\left.\partial \omega\right\}$ permits to conclude.

Lemma 5.2 Let $\psi \in H^{1 / 2}(\partial \omega), \psi_{\varepsilon}(x)=\psi((x-z) / \varepsilon)$ and $w_{\varepsilon} \in H^{1}\left(\Omega_{\varepsilon}\right)$ be the solution of

$$
\left\{\begin{array}{l}
-\Delta w_{\varepsilon}=0 \text { in } \Omega_{\varepsilon} \\
w_{\varepsilon}=0 \text { on } \partial \Omega \\
w_{\varepsilon}=\psi_{\varepsilon} \text { on } \partial \omega_{\varepsilon} .
\end{array}\right.
$$

Let $R>0$ such that $B(z, R) \subset \Omega$. There exists a constant $c>0$ independent of $\varepsilon$ and $\psi$ such that, for $\varepsilon$ small enough,

$$
\begin{gathered}
\left\|w_{\varepsilon}\right\|_{H^{1}\left(\Omega_{\varepsilon}\right)} \leq c\|\psi\|_{H^{1 / 2}(\partial \omega)}, \\
\left\|w_{\varepsilon}\right\|_{H^{1}(\Omega \backslash B(z, R)} \leq \frac{c}{\sqrt{-\log \varepsilon}}\|\psi\|_{H^{1 / 2}(\partial \omega)} .
\end{gathered}
$$

Proof. We assume for convenience of notation that $z=0$.

Step 1 . We denote by $\Psi \in H^{1}(B(0,1) \backslash \bar{\omega})$ a function such that $\Psi=\psi$ on $\partial \omega$ and $\Psi=0$ on $\partial B(0,1)$, obtained from standard lifting, then extended by 0 outside $B(0,1)$. We set $\Psi_{\varepsilon}(x)=\Psi(x / \varepsilon)$ and $\tilde{w}_{\varepsilon}=w_{\varepsilon}-\Psi_{\varepsilon}$. We have from the weak formulation

$$
\int_{\Omega_{\varepsilon}} \nabla w_{\varepsilon} \cdot \nabla \tilde{w}_{\varepsilon} d x=0
$$

whereby

$$
\left\|\nabla \tilde{w}_{\varepsilon}\right\|_{L^{2}\left(\Omega_{\varepsilon}\right)}^{2}=-\int_{\Omega_{\varepsilon}} \nabla \Psi_{\varepsilon} \cdot \nabla \tilde{w}_{\varepsilon} d x .
$$

This entails $\left\|\nabla \tilde{w}_{\varepsilon}\right\|_{L^{2}\left(\Omega_{\varepsilon}\right)} \leq\left\|\nabla \Psi_{\varepsilon}\right\|_{L^{2}\left(\Omega_{\varepsilon}\right)}$ and subsequently $\left\|\nabla w_{\varepsilon}\right\|_{L^{2}\left(\Omega_{\varepsilon}\right)} \leq 2\left\|\nabla \Psi_{\varepsilon}\right\|_{L^{2}\left(\Omega_{\varepsilon}\right)}$. We infer by change of variables

$$
\left\|\nabla w_{\varepsilon}\right\|_{L^{2}\left(\Omega_{\varepsilon}\right)} \leq 2\|\nabla \Psi\|_{L^{2}(B(0,1) \backslash \omega)} \leq c\|\psi\|_{H^{1 / 2}(\partial \omega)} .
$$

We can also lift $\psi$ inside $\omega$ by a function $\tilde{\psi}$, and setting $\tilde{\psi}_{\varepsilon}(x)=\tilde{\psi}(x / \varepsilon)$ we get

$$
\left\|\nabla \tilde{\psi}_{\varepsilon}\right\|_{L^{2}\left(\omega_{\varepsilon}\right)}=\|\nabla \tilde{\psi}\|_{L^{2}(\omega)} \leq c\|\psi\|_{H^{1 / 2}(\partial \omega)} .
$$

Extending $w_{\varepsilon}$ by $\tilde{\psi}_{\varepsilon}$ in $\omega_{\varepsilon}$ and applying the Poincaré inequality in $H_{0}^{1}(\Omega)$ yields

$$
\left\|w_{\varepsilon}\right\|_{H^{1}\left(\Omega_{\varepsilon}\right)} \leq c\|\psi\|_{H^{1 / 2}(\partial \omega)} .
$$

Step 2. To address the second claim we first focus on the problem

$$
\left\{\begin{array}{l}
-\Delta w_{\varepsilon}=0 \text { in } \Omega_{\varepsilon} \\
w_{\varepsilon}=0 \text { on } \partial \Omega \\
w_{\varepsilon}=\bar{\psi} \text { on } \partial \omega_{\varepsilon},
\end{array}\right.
$$

with $\bar{\psi} \in \mathbb{R}$ constant. The variational formulation yields

$$
\left\|\nabla w_{\varepsilon}\right\|_{L^{2}\left(\Omega_{\varepsilon}\right)}^{2} \leq\left\|\nabla v_{\varepsilon}\right\|_{L^{2}\left(\Omega_{\varepsilon}\right)}^{2}
$$


for any $v_{\varepsilon} \in H^{1}\left(\Omega_{\varepsilon}\right)$ such that $v_{\varepsilon}=0$ on $\partial \Omega$ and $v_{\varepsilon}=\bar{\psi}$ on $\partial \omega_{\varepsilon}$. We choose the following one, for some $\rho>0$ such that $B(0, \rho) \subset \Omega$ :

$$
v_{\varepsilon}(x)= \begin{cases}\bar{\psi} & \text { if }|x| \leq \varepsilon \\ \bar{\psi} \frac{\log |x|-\log \rho}{\log \varepsilon-\log \rho} & \text { if } \varepsilon \leq|x| \leq \rho \\ 0 & \text { if }|x| \geq \rho .\end{cases}
$$

This yields

$$
\left\|\nabla v_{\varepsilon}\right\|_{L^{2}\left(\Omega_{\varepsilon}\right)}^{2}=\int_{\varepsilon}^{\rho}\left(\frac{\bar{\psi}}{\log \varepsilon-\log \rho}\right)^{2} \frac{1}{r^{2}} 2 \pi r d r=2 \pi \frac{\bar{\psi}^{2}}{\log \rho-\log \varepsilon} .
$$

It follows that

$$
\left\|\nabla w_{\varepsilon}\right\|_{L^{2}\left(\Omega_{\varepsilon}\right)} \leq\left(\frac{2 \pi}{\log \rho-\log \varepsilon}\right)^{1 / 2}|\bar{\psi}| .
$$

The Poincaré inequality yields for $\varepsilon$ small enough

$$
\left\|w_{\varepsilon}\right\|_{H^{1}\left(\Omega_{\varepsilon}\right)} \leq \frac{c}{\sqrt{-\log \varepsilon}}|\bar{\psi}|
$$

Step 3. We turn to the general case. By lifting, Proposition 5.1 and the Lax-Milgram theorem, there exists a unique $S \in W\left(\mathbb{R}^{2} \backslash \bar{\omega}\right)$ such that $S=\psi$ on $\partial \omega$ and

$$
\int_{\mathbb{R}^{2} \backslash \bar{\omega}} \nabla S \cdot \nabla \Phi d x=0 \quad \forall \Phi \in W_{0}\left(\mathbb{R}^{2} \backslash \bar{\omega}\right) .
$$

Obviously it holds $-\Delta S=0$ in $\mathbb{R}^{2} \backslash \bar{\omega}$ in the sense of distributions. Let $\zeta$ be a smooth function equal to 0 in $B(0,1)$ and 1 outside $B(0,2)$. Set $\hat{S}=\zeta S$ and

$$
G=-\Delta \hat{S}=-\Delta \zeta S-2 \nabla \zeta \cdot \nabla S .
$$

By construction $G$ is supported in the ring $R(0,1,2)$, and it is smooth by elliptic regularity for $S$. Let now $\xi: \mathbb{R}^{2} \rightarrow \mathbb{R}$ be a smooth function equal to 1 in $B(0,2)$ and 0 outside $B(0,3)$ and set $\xi_{\rho}=\xi(x / \rho)$, $\rho>1$. The Green formula yields

$$
\int_{\mathbb{R}^{2}} G d x=\int_{\mathbb{R}^{2}} G \xi_{\rho} d x=\int_{\mathbb{R}^{2}} \nabla \hat{S} \cdot \nabla \xi_{\rho} d x=\int_{\mathbb{R}^{2} \backslash \bar{B}(0,2 \rho)} \nabla \hat{S} \cdot \nabla \xi_{\rho} d x
$$

Applying the Cauchy-Schwarz inequality, using $\nabla \hat{S} \in L^{2}\left(\mathbb{R}^{2}\right)$, a change of variables, and letting $\rho$ go to $+\infty$ results in

$$
\int_{\mathbb{R}^{2}} G d x=0
$$

We have for all $\Phi \in W\left(\mathbb{R}^{2}\right)$, using (24)

$$
\begin{aligned}
\int_{\mathbb{R}^{2}} G \Phi d x & =\int_{\mathbb{R}^{2}} \nabla \zeta \cdot \nabla(S \Phi) d x-2 \int_{\mathbb{R}^{2}} \nabla \zeta \cdot \nabla S \Phi d x \\
& =\int_{\mathbb{R}^{2}} S \nabla \zeta \cdot \nabla \Phi d x-\int_{\mathbb{R}^{2}} \nabla \zeta \cdot \nabla S \Phi d x \\
& =\int_{\mathbb{R}^{2}} \nabla \hat{S} \cdot \nabla \Phi d x-\int_{\mathbb{R}^{2}} \zeta \nabla S \cdot \nabla \Phi d x-\int_{\mathbb{R}^{2}} \nabla \zeta \cdot \nabla S \Phi d x \\
& =\int_{\mathbb{R}^{2}} \nabla \hat{S} \cdot \nabla \Phi d x-\int_{\mathbb{R}^{2}} \nabla S \cdot \nabla(\zeta \Phi) d x .
\end{aligned}
$$


By (23) the latter integral vanishes, resulting in

$$
\int_{\mathbb{R}^{2}} \nabla \hat{S} \cdot \nabla \Phi d x=\int_{\mathbb{R}^{2}} G \Phi d x \quad \forall \Phi \in W\left(\mathbb{R}^{2}\right) .
$$

Let

$$
E(y)=\frac{-1}{2 \pi} \log |y|
$$

be the fundamental solution of the Laplacian and $\hat{S}_{0}=G * E$. Since $G$ is smooth and compactly supported and $E \in L_{\text {loc }}^{1}\left(\mathbb{R}^{2}\right)$, it follows that $\hat{S}_{0}$ is smooth. Using (25) we obtain the expressions

$$
\begin{gathered}
\hat{S}_{0}(x)=\int_{\mathbb{R}^{2}} G(y)(E(x-y)-E(x)) d y \quad \forall x \neq 0, \\
\nabla \hat{S}_{0}(x)=\int_{\mathbb{R}^{2}} G(y)(\nabla E(x-y)-\nabla E(x)) d y \quad \forall x \neq 0 .
\end{gathered}
$$

From the mean value theorem we infer that $\left|\hat{S}_{0}(x)\right| \leq c /|x|$ and $\left|\nabla \hat{S}_{0}(x)\right| \leq c /|x|^{2}$, implying that $\hat{S}_{0} \in W\left(\mathbb{R}^{2}\right)$. Let $\Phi \in W\left(\mathbb{R}^{2}\right)$. We have

$$
\begin{aligned}
\int_{\mathbb{R}^{2}} \nabla \hat{S}_{0} \cdot \nabla \Phi d x=\lim _{\rho \rightarrow+\infty} \int_{\mathbb{R}^{2}} \xi_{\rho} \nabla \hat{S}_{0} \cdot \nabla \Phi d x & =\lim _{\rho \rightarrow+\infty}\left(\int_{\mathbb{R}^{2}} \nabla \hat{S}_{0} \cdot \nabla\left(\xi_{\rho} \Phi\right) d x-\int_{\mathbb{R}^{2}} \nabla \hat{S}_{0} \cdot \nabla \xi_{\rho} \Phi d x\right) \\
& =\int_{\mathbb{R}^{2}} G \Phi d x-\lim _{\rho \rightarrow+\infty} \int_{\mathbb{R}^{2}} \nabla \hat{S}_{0} \cdot \nabla \xi_{\rho} \Phi d x=\int_{\mathbb{R}^{2}} G \Phi d x
\end{aligned}
$$

using the decay properties of $\hat{S}_{0}$ and $\Phi$. Comparing with (26), choosing $\Phi=\hat{S}-\hat{S}_{0}$, we obtain that $\hat{S}=\hat{S}_{0}+\lambda$, for some $\lambda \in \mathbb{R}$. In particular we have the expression

$$
\lambda=-\hat{S}_{0}(0)
$$

showing that $|\lambda| \leq c\|\psi\|_{H^{1 / 2}(\partial \omega)}$. Denoting $S_{0}=S-\lambda$ we have $S_{0}=\hat{S}-\lambda=\hat{S}_{0}$ in $\mathbb{R}^{2} \backslash B(0,2)$, i.e.,

$$
S_{0}(x)=\int_{B(0,2)} G(y) E(x-y) d y \quad \forall x \in \mathbb{R}^{2} \backslash B(0,2) .
$$

We now set

$$
s_{\varepsilon}(x)=S_{0}\left(\frac{x}{\varepsilon}\right) .
$$

Using again (25) we get

$$
s_{\varepsilon}(x)=\int_{B(0,2)} G(y)\left(E\left(\frac{x}{\varepsilon}-y\right)-E\left(\frac{x}{\varepsilon}\right)\right) d y \quad \forall x \in \mathbb{R}^{2} \backslash B(0,2 \varepsilon) .
$$

The particular form of the fundamental solution leads to

$$
s_{\varepsilon}(x)=\int_{B(0,2)} G(y)(E(x-\varepsilon y)-E(x)) d y \quad \forall x \in \mathbb{R}^{2} \backslash B(0,2 \varepsilon) .
$$

The mean value theorem easily shows that

$$
\left\|s_{\varepsilon}\right\|_{H^{1}(\Omega \backslash B(0, R))} \leq c \varepsilon\|G\|_{L^{2}\left(\mathbb{R}^{2}\right)} \leq c \varepsilon\|\psi\|_{H^{1 / 2}(\partial \omega)} .
$$


We note that on $\partial \omega_{\varepsilon}$ we have $s_{\varepsilon}(x)=S_{0}(x / \varepsilon)=S(x / \varepsilon)-\lambda=\psi(x / \varepsilon)-\lambda$. We now define $r_{\varepsilon}=w_{\varepsilon}-s_{\varepsilon}$, solution of

$$
\left\{\begin{array}{l}
-\Delta r_{\varepsilon}=0 \text { in } \Omega_{\varepsilon} \\
r_{\varepsilon}=-s_{\varepsilon} \text { on } \partial \Omega \\
r_{\varepsilon}=\lambda \text { on } \partial \omega_{\varepsilon} .
\end{array}\right.
$$

We have by step 2 and a standard decomposition

$$
\left\|r_{\varepsilon}\right\|_{H^{1}\left(\Omega_{\varepsilon}\right)} \leq c\left\|s_{\varepsilon}\right\|_{H^{1 / 2}(\partial \Omega)}+c \frac{|\lambda|}{\sqrt{-\log \varepsilon}} \leq \frac{c}{\sqrt{-\log \varepsilon}}\|\psi\|_{H^{1 / 2}(\partial \omega)} .
$$

This completes the proof by $w_{\varepsilon}=s_{\varepsilon}+r_{\varepsilon}$.

\subsection{Variation of the direct state}

Set $\tilde{u}_{\varepsilon}=u_{\varepsilon}-u_{0}$. It solves

$$
\left\{\begin{array}{l}
-\Delta \tilde{u}_{\varepsilon}=0 \text { in } \Omega_{\varepsilon} \\
\tilde{u}_{\varepsilon}=0 \text { on } \partial \Omega \\
\tilde{u}_{\varepsilon}=-u_{0} \text { on } \partial \omega_{\varepsilon} .
\end{array}\right.
$$

We define $h_{\varepsilon} \in \mathcal{C}^{\infty}\left(\mathbb{R}^{2} \backslash\{z\}\right)$ and $r_{\varepsilon} \in H^{1}(\Omega)$ by

$$
h_{\varepsilon}(x)=-\frac{\log |x-z|}{\log \varepsilon} u_{0}(z), \quad\left\{\begin{array}{l}
-\Delta r_{\varepsilon}=0 \text { in } \Omega \\
r_{\varepsilon}=-h_{\varepsilon} \text { on } \partial \Omega .
\end{array}\right.
$$

As for the fundamental solution we have $\Delta h_{\varepsilon}=0$ in $\mathbb{R}^{2} \backslash\{z\}$. We now set $e_{\varepsilon}=\tilde{u}_{\varepsilon}-h_{\varepsilon}-r_{\varepsilon}$, which solves

$$
\left\{\begin{array}{l}
-\Delta e_{\varepsilon}=0 \text { in } \Omega_{\varepsilon} \\
e_{\varepsilon}=0 \text { on } \partial \Omega \\
e_{\varepsilon}=-u_{0}-h_{\varepsilon}-r_{\varepsilon} \text { on } \partial \omega_{\varepsilon}
\end{array}\right.
$$

Lemma 5.3 If $u_{0}$ is $\mathcal{C}^{1}$ is a neighborhood of $z$ then

$$
\begin{gathered}
\left\|r_{\varepsilon}\right\|_{H^{1}(\Omega)}=O\left((-\log \varepsilon)^{-1}\right), \\
\left\|e_{\varepsilon}\right\|_{H^{1}(\Omega \backslash B(z, R))}=O\left((-\log \varepsilon)^{-3 / 2}\right) .
\end{gathered}
$$

Proof. The first estimate is obvious since $\left\|h_{\varepsilon}\right\|_{H^{1 / 2}(\partial \Omega)}=O\left((-\log \varepsilon)^{-1}\right)$ by construction.

Set

$$
\psi_{\varepsilon}(x)=\left(-u_{0}-h_{\varepsilon}-r_{\varepsilon}\right)(z+\varepsilon x) .
$$

We decompose as

$$
\psi_{\varepsilon}(x)=[\underbrace{u_{0}(z)-u_{0}(z+\varepsilon x)}_{p_{\varepsilon}(x)}]-[\underbrace{u_{0}(z)+h_{\varepsilon}(z+\varepsilon x)}_{q_{\varepsilon}(x)}]-\underbrace{r_{\varepsilon}(z+\varepsilon x)}_{\hat{r}_{\varepsilon}(x)} .
$$

By regularity of $u_{0}$ we have immediately $\left\|p_{\varepsilon}\right\|_{H^{1 / 2}(\partial \omega)}=O(\varepsilon)$. Next, from

$$
q_{\varepsilon}(x)=u_{0}(z)\left(1-\frac{\log |\varepsilon x|}{\log \varepsilon}\right)=-u_{0}(z) \frac{\log |x|}{\log \varepsilon},
$$

we get $\left\|q_{\varepsilon}\right\|_{H^{1 / 2}(\partial \omega)}=O\left((-\log \varepsilon)^{-1}\right)$. Lastly, a change of variables yields

$$
\left\|\hat{r}_{\varepsilon}\right\|_{H^{1}(\omega)} \leq\left\|\nabla r_{\varepsilon}\right\|_{L^{2}\left(\omega_{\varepsilon}\right)}+\varepsilon^{-1}\left\|r_{\varepsilon}\right\|_{L^{2}\left(\omega_{\varepsilon}\right)} \leq\left\|\nabla r_{\varepsilon}\right\|_{L^{2}\left(\omega_{\varepsilon}\right)}+c\left\|r_{\varepsilon}\right\|_{L^{\infty}\left(\omega_{\varepsilon}\right)} .
$$

By elliptic regularity we have $\left\|\nabla r_{\varepsilon}\right\|_{L^{2}\left(\omega_{\varepsilon}\right)}+\left\|r_{\varepsilon}\right\|_{L^{\infty}\left(\omega_{\varepsilon}\right)} \leq c\left\|h_{\varepsilon}\right\|_{H^{1 / 2}(\partial \Omega)}$, thus $\left\|\hat{r}_{\varepsilon}\right\|_{H^{1 / 2}(\partial \omega)}=O\left((-\log \varepsilon)^{-1}\right)$. We conclude using Lemma 5.2.

We infer from Lemma 5.3 and the triangle inequality: 
Lemma 5.4 If $u_{0}$ is $\mathcal{C}^{1}$ is a neighborhood of $z$ then

$$
\left\|\tilde{u}_{\varepsilon}\right\|_{H^{1}(\Omega \backslash B(z, R)}=O\left((-\log \varepsilon)^{-1}\right) .
$$

\subsection{Variation of the adjoint state}

In view of Proposition 3.1 and (20)-(21), we define the adjoint state $v_{\varepsilon} \in H_{0}^{1}(\Omega)$ solution of

$$
\int_{\Omega} \nabla v_{\varepsilon} \cdot \nabla \varphi d x=-\int_{0}^{1} d J_{\varepsilon}\left(t u_{\varepsilon}+(1-t) u_{0}\right) \varphi d t \quad \forall \varphi \in H_{0}^{1}(\Omega) .
$$

In particular the unperturbed adjoint state $v_{0}$ satisfies

$$
\int_{\Omega} \nabla v_{0} \cdot \nabla \varphi d x=-d J_{0}\left(u_{0}\right) \varphi \quad \forall \varphi \in H_{0}^{1}(\Omega)
$$

Lemma 5.5 If $u_{0}$ is $\mathcal{C}^{1}$ is a neighborhood of $z$ then

$$
\left\|v_{\varepsilon}-v_{0}\right\|_{H^{1}(\Omega)}=O\left((-\log \varepsilon)^{-1}\right) .
$$

Proof. Set $\tilde{v}_{\varepsilon}=v_{\varepsilon}-v_{0}$. We have

$$
\int_{\Omega} \nabla \tilde{v}_{\varepsilon} \cdot \nabla \varphi d x=\int_{0}^{1}\left(d J_{0}\left(u_{0}\right)-d J_{\varepsilon}\left(t u_{\varepsilon}+(1-t) u_{0}\right)\right) \varphi d t \quad \forall \varphi \in H_{0}^{1}(\Omega),
$$

leading to

$$
\int_{\Omega} \nabla \tilde{v}_{\varepsilon} \cdot \nabla \varphi d x=\int_{0}^{1}\left(d \hat{J}\left(u_{0 \mid \hat{\Omega}}\right)-d \hat{J}\left(\left(t u_{\varepsilon}+(1-t) u_{0}\right)_{\mid \hat{\Omega}}\right) \varphi_{\mid \hat{\Omega}} d t \quad \forall \varphi \in H_{0}^{1}(\Omega) .\right.
$$

Choosing $\varphi=\tilde{v}_{\varepsilon}$ and using that $d \hat{J}$ is Lipschitz yields

$$
\left\|\tilde{v}_{\varepsilon}\right\|_{H^{1}(\Omega)} \leq c\left\|\tilde{u}_{\varepsilon}\right\|_{H^{1}(\hat{\Omega})} .
$$

The conclusion follows from Lemma 5.4.

\subsection{Variation of the Lagrangian}

In view of Proposition 3.1 we define the Lagrangian

$$
\mathcal{L}_{\varepsilon}(u, v)=J_{\varepsilon}(u)+\int_{\Omega} \nabla u \cdot \nabla v d x-\int_{\Omega} f v+\int_{\partial \omega_{\varepsilon}} \frac{\partial u_{\varepsilon}}{\partial n} v d s .
$$

This provides the variation

$$
\left(\mathcal{L}_{\varepsilon}-\mathcal{L}_{0}\right)\left(u_{0}, v_{\varepsilon}\right)=\int_{\partial \omega_{\varepsilon}} \frac{\partial u_{\varepsilon}}{\partial n} v_{\varepsilon} d s
$$

Lemma 5.6 If $u_{0}, v_{0}$ are of class $\mathcal{C}^{1}$ in a neighborhood of $z$ then

$$
\left.\left(\mathcal{L}_{\varepsilon}-\mathcal{L}_{0}\right)\left(u_{0}, v_{\varepsilon}\right)=\int_{\partial \omega_{\varepsilon}} \frac{\partial \tilde{u}_{\varepsilon}}{\partial n} v_{0} d s+O\left((-\log \varepsilon)^{-2}\right)\right)
$$


ProOF. We work with the decomposition

$$
\begin{aligned}
\left(\mathcal{L}_{\varepsilon}-\mathcal{L}_{0}\right)\left(u_{0}, v_{\varepsilon}\right)-\int_{\partial \omega_{\varepsilon}} \frac{\partial \tilde{u}_{\varepsilon}}{\partial n} v_{0} d s & =\int_{\partial \omega_{\varepsilon}} \frac{\partial u_{0}}{\partial n} v_{\varepsilon} d s+\int_{\partial \omega_{\varepsilon}} \frac{\partial \tilde{u}_{\varepsilon}}{\partial n}\left(v_{\varepsilon}-v_{0}\right) d s \\
& =\int_{\omega_{\varepsilon}} \nabla u_{0} \cdot \nabla v_{\varepsilon} d x-\int_{\Omega_{\varepsilon}} \nabla \tilde{u}_{\varepsilon} \cdot \nabla\left(v_{\varepsilon}-v_{0}\right) d x
\end{aligned}
$$

Extending $\tilde{u}_{\varepsilon}$ by $-u_{0}$ in $\omega_{\varepsilon}$ allows to write

$$
\begin{aligned}
\left(\mathcal{L}_{\varepsilon}-\mathcal{L}_{0}\right)\left(u_{0}, v_{\varepsilon}\right)-\int_{\partial \omega_{\varepsilon}} \frac{\partial \tilde{u}_{\varepsilon}}{\partial n} v_{0} d s= & -\int_{\Omega} \nabla \tilde{u}_{\varepsilon} \cdot \nabla\left(v_{\varepsilon}-v_{0}\right) d x+\int_{\omega_{\varepsilon}} \nabla u_{0} \cdot \nabla v_{0} d x \\
= & \int_{0}^{1}\left(d \hat{J}\left(\left(t u_{\varepsilon}+(1-t) u_{0}\right)_{\mid \hat{\Omega}}\right)-d \hat{J}\left(u_{0 \mid \hat{\Omega}}\right)\right)\left(\tilde{u}_{\varepsilon}\right)_{\mid \hat{\Omega}} d t \\
& +\int_{\omega_{\varepsilon}} \nabla u_{0} \cdot \nabla v_{0} d x,
\end{aligned}
$$

where the last equality is obtained as in Lemma 5.5. We conclude using Lemma 5.4.

Lemma 5.7 If $u_{0}, v_{0}$ are of class $\mathcal{C}^{1}$ in a neighborhood of $z$ then

$$
\left(\mathcal{L}_{\varepsilon}-\mathcal{L}_{0}\right)\left(u_{0}, v_{\varepsilon}\right)=\frac{2 \pi}{-\log \varepsilon} u_{0}(z) v_{0}(z)+O\left((-\log \varepsilon)^{-3 / 2}\right) .
$$

Proof. We decompose the expression found in Lemma 5.6 as

$$
\begin{aligned}
\left(\mathcal{L}_{\varepsilon}-\mathcal{L}_{0}\right)\left(u_{0}, v_{\varepsilon}\right)= & \int_{\partial \omega_{\varepsilon}} \frac{\partial h_{\varepsilon}}{\partial n} v_{0} d s+\int_{\partial \omega_{\varepsilon}} \frac{\partial r_{\varepsilon}}{\partial n} v_{0} d s+\int_{\partial \omega_{\varepsilon}} \frac{\partial e_{\varepsilon}}{\partial n} v_{0} d s+O\left((-\log \varepsilon)^{-2}\right) \\
= & \int_{\partial \omega_{\varepsilon}} \frac{\partial h_{\varepsilon}}{\partial n} v_{0}(z) d s+\int_{\partial \omega_{\varepsilon}} \frac{\partial h_{\varepsilon}}{\partial n}\left(v_{0}-v_{0}(z)\right) d s \\
& +\int_{\omega_{\varepsilon}} \nabla r_{\varepsilon} \cdot \nabla v_{0} d x-\int_{\Omega_{\varepsilon}} \nabla e_{\varepsilon} \cdot \nabla v_{0} d x+O\left((-\log \varepsilon)^{-2}\right) .
\end{aligned}
$$

Let $\rho>0$ such that $B(0, \rho) \subset \omega$. We define

$$
\tilde{h}_{\varepsilon}(x)= \begin{cases}h_{\varepsilon}(x) & \text { if }|x-z| \geq \rho \varepsilon \\ -\left(\frac{\log \rho}{\log \varepsilon}+1\right) u_{0}(z) & \text { if }|x-z| \leq \rho \varepsilon .\end{cases}
$$

This truncation ensures that $\tilde{h}_{\varepsilon} \in H^{1}(\Omega)$. We extend $e_{\varepsilon}$ by $-u_{0}-\tilde{h}_{\varepsilon}-r_{\varepsilon}$ in $\omega_{\varepsilon}$ to write

$$
\begin{aligned}
\left(\mathcal{L}_{\varepsilon}-\mathcal{L}_{0}\right)\left(u_{0}, v_{\varepsilon}\right)= & \int_{\partial \omega_{\varepsilon}} \frac{\partial h_{\varepsilon}}{\partial n} v_{0}(z) d s+\int_{\partial \omega_{\varepsilon}} \frac{\partial h_{\varepsilon}}{\partial n}\left(v_{0}-v_{0}(z)\right) d s \\
& -\int_{\Omega} \nabla e_{\varepsilon} \cdot \nabla v_{0} d x-\int_{\omega_{\varepsilon}} \nabla\left(\tilde{h}_{\varepsilon}+u_{0}\right) \cdot \nabla v_{0} d x+O\left((-\log \varepsilon)^{-2}\right) \\
= & \int_{\partial \omega_{\varepsilon}} \frac{\partial h_{\varepsilon}}{\partial n} v_{0}(z) d s+\int_{\partial \omega_{\varepsilon}} \frac{\partial h_{\varepsilon}}{\partial n}\left(v_{0}-v_{0}(z)\right) d s \\
& +d \hat{J}\left(u_{0 \mid \hat{\Omega}}\right) e_{\varepsilon \mid \hat{\Omega}}-\int_{\omega_{\varepsilon}} \nabla\left(\tilde{h}_{\varepsilon}+u_{0}\right) \cdot \nabla v_{0} d x+O\left((-\log \varepsilon)^{-2}\right) .
\end{aligned}
$$

By the definition of $h_{\varepsilon}$, Lemma 5.3 and the smoothness assumptions we arrive at

$$
\left(\mathcal{L}_{\varepsilon}-\mathcal{L}_{0}\right)\left(u_{0}, v_{\varepsilon}\right)=\int_{\partial \omega_{\varepsilon}} \frac{\partial h_{\varepsilon}}{\partial n} v_{0}(z) d s+O\left((-\log \varepsilon)^{-3 / 2}\right) .
$$


The Green formula yields

$$
\int_{\partial \omega_{\varepsilon}} \frac{\partial h_{\varepsilon}}{\partial n} d s=\int_{\partial B(z, \varepsilon)} \frac{\partial h_{\varepsilon}}{\partial n} d s=\frac{-2 \pi}{\log \varepsilon} u_{0}(z),
$$

which completes the proof.

\subsection{Expression of the topological asymptotic expansion}

Based on the previous results we arrive at the following counterpart to Theorem 4.9.

Theorem 5.8 Consider a cost function of form (22). Let $v_{0} \in H_{0}^{1}(\Omega)$ be the solution of (29). Then

$$
J_{\varepsilon}\left(u_{\varepsilon}\right)-J_{0}\left(u_{0}\right)=\frac{2 \pi}{-\log \varepsilon} u_{0}(z) v_{0}(z)+o\left(\frac{1}{-\log \varepsilon}\right) .
$$

We observe that this expression does not depend on the shape of the hole. This is typical of the $2 \mathrm{D}$ case. We infer the topological derivative

$$
d_{T} \mathcal{J}(\Omega, \omega, z)=u_{0}(z) v_{0}(z) .
$$

Lastly, we highlight in Fig. 2 the slow convergence of the cost function compared with the inclusion case by plotting the functions $f_{1}(\varepsilon)=\frac{1}{-\log \varepsilon}$ and $f_{2}(\varepsilon)=\varepsilon^{2}$ appearing in Theorems 5.8 and 4.9 in $2 \mathrm{D}$, respectively.

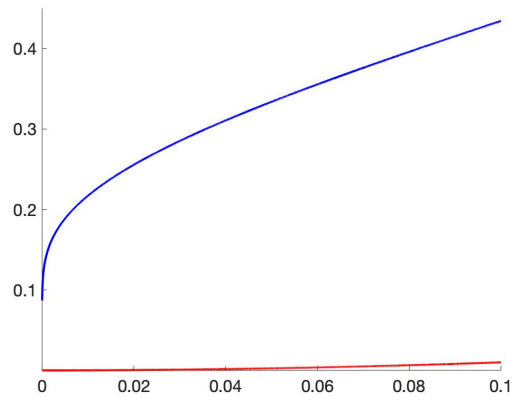

Figure 2: Comparison of the functions $f_{1}(\varepsilon)=\frac{1}{-\log \varepsilon}$ (in blue) and $f_{2}(\varepsilon)=\varepsilon^{2}$ (in red).

\section{Dirichlet case in 3D}

We consider the same problem as in section 5.1, but now in dimension $N=3$. We follow the same general scheme, but significant technical differences will show up.

\subsection{Preliminary estimate}

The weighted Sobolev spaces $W\left(\mathbb{R}^{3}\right)$ and $W_{0}\left(\mathbb{R}^{3}\right)$ are defined in the same way as their $2 \mathrm{D}$ counterparts, with the weight function

$$
w(x)=\frac{1}{\sqrt{1+|x|^{2}}} .
$$

It leads to the same Poincaré inequality [Dautray and Lions, 1988, Amrouche et al., 1994]:

$$
\|u\|_{W\left(\mathbb{R}^{3} \backslash \bar{\omega}\right)} \leq c\|\nabla u\|_{L^{2}\left(\mathbb{R}^{3} \backslash \bar{\omega}\right)} \quad \forall u \in W_{0}\left(\mathbb{R}^{3} \backslash \bar{\omega}\right) .
$$


Lemma 6.1 Let $\psi \in H^{1 / 2}(\partial \omega), \psi_{\varepsilon}(x)=\psi((x-z) / \varepsilon)$ and $w_{\varepsilon} \in H^{1}\left(\Omega_{\varepsilon}\right)$ be the solution of

$$
\left\{\begin{array}{l}
-\Delta w_{\varepsilon}=0 \text { in } \Omega_{\varepsilon} \\
w_{\varepsilon}=0 \text { on } \partial \Omega \\
w_{\varepsilon}=\psi_{\varepsilon} \text { on } \partial \omega_{\varepsilon} .
\end{array}\right.
$$

Let $R>0$ such that $B(z, R) \subset \Omega$. There exists a constant $c>0$ independent of $\varepsilon$ and $\psi$ such that, for $\varepsilon$ small enough,

$$
\begin{gathered}
\left\|w_{\varepsilon}\right\|_{H^{1}\left(\Omega_{\varepsilon}\right)} \leq c \sqrt{\varepsilon}\|\psi\|_{H^{1 / 2}(\partial \omega)}, \\
\left\|w_{\varepsilon}\right\|_{H^{1}(\Omega \backslash B(z, R)} \leq c \varepsilon\|\psi\|_{H^{1 / 2}(\partial \omega)} .
\end{gathered}
$$

Proof. We assume here again for simplicity that $z=0$. The proof of (32) follows exactly the same lines as in Lemma 5.2, hence we focus on (33). By lifting, (31) and the Lax-Milgram theorem, there exists a unique $S \in W\left(\mathbb{R}^{3} \backslash \bar{\omega}\right)$ such that $S=\psi$ on $\partial \omega$ and

$$
\int_{\mathbb{R}^{3} \backslash \bar{\omega}} \nabla S \cdot \nabla \Phi d x=0 \quad \forall \Phi \in W_{0}\left(\mathbb{R}^{3} \backslash \bar{\omega}\right) .
$$

Similarly to Lemma 5.2 we introduce a smooth function $\zeta$ equal to 0 in $B(0,1)$ and 1 outside $B(0,2)$, we set $\hat{S}=\zeta S$ and $\hat{S}_{0}=G * E$, with $G=-\Delta \hat{S}$,

$$
E(y)=\frac{1}{4 \pi|y|}
$$

the fundamental solution of the Laplacian. Here we do not have the counterpart to (25), nevertheless the definition directly shows that $\hat{S}_{0} \in W\left(\mathbb{R}^{3}\right)$. It follows by the same argument as in Lemma 5.2 that $\hat{S}=\hat{S}_{0}+\lambda$, for some $\lambda \in \mathbb{R}$, but here the inclusion $\hat{S}-\hat{S}_{0} \in W\left(\mathbb{R}^{3}\right)$ implies $\lambda=0$ since constants do not belong to $W\left(\mathbb{R}^{3}\right)$. We arrive at

$$
S(x)=\int_{B(0,2)} G(y) E(x-y) d y \quad \forall x \in \mathbb{R}^{3} \backslash B(0,2) .
$$

Setting

$$
s_{\varepsilon}(x)=S\left(\frac{x}{\varepsilon}\right),
$$

we get

$$
s_{\varepsilon}(x)=\varepsilon \int_{B(0,2)} G(y) E(x-\varepsilon y) d y \quad \forall x \in \mathbb{R}^{3} \backslash B(0,2 \varepsilon) .
$$

This representation entails

$$
\left\|s_{\varepsilon}\right\|_{H^{1}(\Omega \backslash B(0, R))} \leq c \varepsilon\|G\|_{L^{2}\left(\mathbb{R}^{3}\right)} \leq c \varepsilon\|\psi\|_{H^{1 / 2}(\partial \omega)} .
$$

We now define $r_{\varepsilon}=w_{\varepsilon}-s_{\varepsilon}$, which is solution of

$$
\left\{\begin{array}{l}
-\Delta r_{\varepsilon}=0 \text { in } \Omega_{\varepsilon} \\
r_{\varepsilon}=-s_{\varepsilon} \text { on } \partial \Omega \\
r_{\varepsilon}=0 \text { on } \partial \omega_{\varepsilon} .
\end{array}\right.
$$

The decomposition $w_{\varepsilon}=s_{\varepsilon}+r_{\varepsilon}$ combined with (36) yields (33). 


\subsection{Variation of the direct state}

Similarly to section 5.3 we set $\tilde{u}_{\varepsilon}=u_{\varepsilon}-u_{0}$, which solves

$$
\left\{\begin{array}{l}
-\Delta \tilde{u}_{\varepsilon}=0 \text { in } \Omega_{\varepsilon} \\
\tilde{u}_{\varepsilon}=0 \text { on } \partial \Omega \\
\tilde{u}_{\varepsilon}=-u_{0} \text { on } \partial \omega_{\varepsilon} .
\end{array}\right.
$$

Now we consider the reference problem: find $U \in W\left(\mathbb{R}^{3} \backslash \bar{\omega}\right)$ such that

$$
\left\{\begin{array}{l}
-\Delta U=0 \text { in } \mathbb{R}^{3} \backslash \bar{\omega} \\
U=1 \text { on } \partial \omega
\end{array}\right.
$$

whose variational formulation is as in (34). We define $h_{\varepsilon} \in W\left(\mathbb{R}^{3} \backslash \bar{\omega}_{\varepsilon}\right)$ and $r_{\varepsilon} \in H^{1}(\Omega)$ by

$$
h_{\varepsilon}(x)=-u_{0}(z) U\left(\frac{x-z}{\varepsilon}\right), \quad\left\{\begin{array}{l}
-\Delta r_{\varepsilon}=0 \text { in } \Omega \\
r_{\varepsilon}=-h_{\varepsilon} \text { on } \partial \Omega .
\end{array}\right.
$$

We now set $e_{\varepsilon}=\tilde{u}_{\varepsilon}-h_{\varepsilon}-r_{\varepsilon}$. It solves

$$
\left\{\begin{array}{l}
-\Delta e_{\varepsilon}=0 \text { in } \Omega_{\varepsilon} \\
e_{\varepsilon}=0 \text { on } \partial \Omega \\
e_{\varepsilon}=-u_{0}-h_{\varepsilon}-r_{\varepsilon} \text { on } \partial \omega_{\varepsilon} .
\end{array}\right.
$$

Lemma 6.2 If $u_{0}$ is $\mathcal{C}^{1}$ is a neighborhood of $z$ then

$$
\begin{gathered}
\left\|h_{\varepsilon}\right\|_{H^{1}\left(\Omega_{\varepsilon} \backslash B(z, R)\right)}=O(\varepsilon), \\
\left\|r_{\varepsilon}\right\|_{H^{1}(\Omega)}=O(\varepsilon), \\
\left\|e_{\varepsilon}\right\|_{H^{1}(\Omega \backslash B(z, R))}=O\left(\varepsilon^{3 / 2}\right) .
\end{gathered}
$$

ProOF. The same representation as in (35) shows that $|U(x)| \leq c /|x|$ and $|\nabla U(x)| \leq c /|x|^{2}$, hence $\left\|h_{\varepsilon}\right\|_{H^{1}(\Omega \backslash B(z, R))}=O(\varepsilon)$, as in (36) whereby also $\left\|r_{\varepsilon}\right\|_{H^{1}(\Omega)}=O(\varepsilon)$. Set $\hat{r}_{\varepsilon}(x)=r_{\varepsilon}(z+\varepsilon x)$. A change of variables yields

$$
\left\|\hat{r}_{\varepsilon}\right\|_{H^{1}(\omega)} \leq \varepsilon^{-1 / 2}\left\|\nabla r_{\varepsilon}\right\|_{L^{2}\left(\omega_{\varepsilon}\right)}+\varepsilon^{-3 / 2}\left\|r_{\varepsilon}\right\|_{L^{2}\left(\omega_{\varepsilon}\right)} \leq \varepsilon^{-1 / 2}\left\|\nabla r_{\varepsilon}\right\|_{L^{2}\left(\omega_{\varepsilon}\right)}+c \varepsilon^{-1 / 2}\left\|r_{\varepsilon}\right\|_{L^{6}(\Omega)}=O\left(\varepsilon^{1 / 2}\right),
$$

where we have used the Sobolev embedding $H^{1} \hookrightarrow L^{6}$. We obtain $\left\|\left(u_{0}+h_{\varepsilon}+r_{\varepsilon}\right)(z+\varepsilon x)\right\|_{H^{1 / 2}(\partial \omega)}=$ $\|\left(u_{0}(z+\varepsilon x)-u_{0}(z)+\hat{r}_{\varepsilon}(x) \|_{H^{1 / 2}(\partial \omega)}=O\left(\varepsilon^{1 / 2}\right)\right.$. We conclude by Lemma 6.1.

We deduce:

Lemma 6.3 If $u_{0}$ is $\mathcal{C}^{1}$ is a neighborhood of $z$ then

$$
\left\|\tilde{u}_{\varepsilon}\right\|_{H^{1}(\Omega \backslash B(z, R)}=O(\varepsilon) .
$$

\subsection{Variation of the adjoint state}

We again define the adjoint state $v_{\varepsilon} \in H_{0}^{1}(\Omega)$ solution of

$$
\int_{\Omega} \nabla v_{\varepsilon} \cdot \nabla \varphi d x=-\int_{0}^{1} d J_{\varepsilon}\left(t u_{\varepsilon}+(1-t) u_{0}\right) \varphi d t \quad \forall \varphi \in H_{0}^{1}(\Omega) .
$$

Similarly to Lemma 5.5 we have:

Lemma 6.4 If $u_{0}$ is $\mathcal{C}^{1}$ is a neighborhood of $z$ then

$$
\left\|v_{\varepsilon}-v_{0}\right\|_{H^{1}(\Omega)}=O(\varepsilon)
$$




\subsection{Variation of the Lagrangian}

We consider the same Lagrangian as in (30).

Lemma 6.5 If $u_{0}, v_{0}$ are of class $\mathcal{C}^{1}$ in a neighborhood of $z$ then

$$
\left(\mathcal{L}_{\varepsilon}-\mathcal{L}_{0}\right)\left(u_{0}, v_{\varepsilon}\right)=\int_{\partial \omega_{\varepsilon}} \frac{\partial \tilde{u}_{\varepsilon}}{\partial n} v_{0} d s+O\left(\varepsilon^{2}\right)
$$

Proof. Like in Lemma 5.6 we have

$\left(\mathcal{L}_{\varepsilon}-\mathcal{L}_{0}\right)\left(u_{0}, v_{\varepsilon}\right)-\int_{\partial \omega_{\varepsilon}} \frac{\partial \tilde{u}_{\varepsilon}}{\partial n} v_{0} d s=\int_{0}^{1}\left(d \hat{J}\left(\left(t u_{\varepsilon}+(1-t) u_{0}\right)_{\mid \hat{\Omega}}\right)-d \hat{J}\left(u_{0 \mid \hat{\Omega}}\right)\right)\left(\tilde{u}_{\varepsilon}\right)_{\mid \hat{\Omega}} d t+\int_{\omega_{\varepsilon}} \nabla u_{0} \cdot \nabla v_{0} d x$.

We conclude using Lemma 6.3.

The standard capacity of $\omega$ can be defined by (see e.g. [Henrot and Pierre, 2018])

$$
\mathcal{K}=-\int_{\partial \omega} \frac{\partial U}{\partial n} d s=\int_{\mathbb{R}^{3} \backslash \omega}|\nabla U|^{2} d x .
$$

Lemma 6.6 If $u_{0}, v_{0}$ are of class $\mathcal{C}^{1}$ in a neighborhood of $z$ then

$$
\left(\mathcal{L}_{\varepsilon}-\mathcal{L}_{0}\right)\left(u_{0}, v_{\varepsilon}\right)=\mathcal{K} \varepsilon u_{0}(z) v_{0}(z)+O\left(\varepsilon^{3 / 2}\right) .
$$

Proof. We decompose the expression found in Lemma 6.5 as

$\left(\mathcal{L}_{\varepsilon}-\mathcal{L}_{0}\right)\left(u_{0}, v_{\varepsilon}\right)=\int_{\partial \omega_{\varepsilon}} \frac{\partial h_{\varepsilon}}{\partial n} v_{0}(z) d s+\int_{\partial \omega_{\varepsilon}} \frac{\partial h_{\varepsilon}}{\partial n}\left(v_{0}-v_{0}(z)\right) d s+\int_{\omega_{\varepsilon}} \nabla r_{\varepsilon} \cdot \nabla v_{0} d x-\int_{\Omega_{\varepsilon}} \nabla e_{\varepsilon} \cdot \nabla v_{0} d x+O\left(\varepsilon^{2}\right)$.

Inside $\omega_{\varepsilon}$ we extend $h_{\varepsilon}$ by $-u_{0}(z)$ and $e_{\varepsilon}$ by $-u_{0}+u_{0}(z)-r_{\varepsilon}$. This permits to write

$$
\begin{aligned}
\left(\mathcal{L}_{\varepsilon}-\mathcal{L}_{0}\right)\left(u_{0}, v_{\varepsilon}\right)= & \int_{\partial \omega_{\varepsilon}} \frac{\partial h_{\varepsilon}}{\partial n} v_{0}(z) d s+\int_{\partial \omega_{\varepsilon}} \frac{\partial h_{\varepsilon}}{\partial n}\left(v_{0}-v_{0}(z)\right) d s \\
& -\int_{\Omega} \nabla e_{\varepsilon} \cdot \nabla v_{0} d x-\int_{\omega_{\varepsilon}} \nabla u_{0} \cdot \nabla v_{0} d x+O\left(\varepsilon^{2}\right) \\
= & \int_{\partial \omega_{\varepsilon}} \frac{\partial h_{\varepsilon}}{\partial n} v_{0}(z) d s+\int_{\partial \omega_{\varepsilon}} \frac{\partial h_{\varepsilon}}{\partial n}\left(v_{0}-v_{0}(z)\right) d s \\
& +d \hat{J}\left(u_{0 \mid \hat{\Omega}}\right) e_{\varepsilon \mid \hat{\Omega}}-\int_{\omega_{\varepsilon}} \nabla u_{0} \cdot \nabla v_{0} d x+O\left(\varepsilon^{2}\right) .
\end{aligned}
$$

The claim follows from the definition of $h_{\varepsilon}$ and Lemma 6.2.

\subsection{Expression of the topological asymptotic expansion}

From the preceding findings we infer the 3D counterpart to Theorem 5.8.

Theorem 6.7 Consider a cost function of form (22). Let $v_{0} \in H_{0}^{1}(\Omega)$ be the solution of (29), $\mathcal{K}$ be defined by (40). Then

$$
J_{\varepsilon}\left(u_{\varepsilon}\right)-J_{0}\left(u_{0}\right)=\mathcal{K} \varepsilon u_{0}(z) v_{0}(z)+o(\varepsilon) .
$$

We note that here, unlike in the $2 \mathrm{D}$ case, the expression of the topological asymptotic expansion depends on the shape of the hole, through the capacity $\mathcal{K}$. In the case of the ball $\omega=B(0,1)$ the solution to (38) is found as

$$
U(x)=\frac{1}{|x|}
$$

leading to the capacity $\mathcal{K}=4 \pi$.

Remark 6.8 Like in the inclusion case, more general cost functions may yield additional terms, see e.g. [Guillaume and Sid Idris, 2002, Amstutz, 2006b]. 


\subsection{Extensions}

Topological derivatives in the Dirichlet case have been comparatively less studied than in the inclusion / Neumann cases. Related contributions can be found in particular on the linear elasticity case [Garreau et al., 2001], the Helmholtz equation [Samet et al., 2003], the Stokes problem [Guillaume and Sid Idris, 2004], and semilinear problems [Amstutz, 2006b, Iguernane et al., 2009].

\section{Conclusion}

Some introductory mathematical elements on the topological derivative concept have been thoroughly presented. They may help the reader to enter into the more advanced literature and tackle further problems on this field under active development.

\section{Acknowledgements}

The author benefited from the supports of the chair "Modeling advanced polymers for innovative material solutions" led by the Ecole polytechnique and the Fondation de l'Ecole polytechnique and sponsored by Arkema, and of the project ANR-18-CE40-0013 SHAPO financed by the Agence Nationale de la Recherche.

\section{References}

[Allaire, 2007] Allaire, G. (2007). Conception optimale de structures, volume 58 of Mathématiques E Applications (Berlin) [Mathematics 85 Applications]. Springer-Verlag, Berlin. With the collaboration of Marc Schoenauer (INRIA) in the writing of Chapter 8.

[Ammari and Kang, 2007] Ammari, H. and Kang, H. (2007). Polarization and moment tensors, volume 162 of Applied Mathematical Sciences. Springer, New York.

[Amrouche et al., 1994] Amrouche, C., Girault, V., and Giroire, J. (1994). Weighted Sobolev spaces for Laplace's equation in $\mathbf{R}^{n}$. J. Math. Pures Appl. (9), 73(6):579-606.

[Amstutz, 2003] Amstutz, S. (2003). Aspects théoriques et numériques en optimisation de forme topologique. PhD thesis, Institut National des Sciences Appliquées, Toulouse, France.

[Amstutz, 2006a] Amstutz, S. (2006a). Sensitivity analysis with respect to a local perturbation of the material property. Asymptot. Anal., 49(1-2):87-108.

[Amstutz, 2006b] Amstutz, S. (2006b). Topological sensitivity analysis for some nonlinear PDE system. J. Math. Pures Appl. (9), 85(4):540-557.

[Amstutz and Bonnafé, 2017] Amstutz, S. and Bonnafé, A. (2017). Topological derivatives for a class of quasilinear elliptic equations. J. Math. Pures Appl. (9), 107(4):367-408.

[Amstutz and Dominguez, 2008] Amstutz, S. and Dominguez, N. (2008). Topological sensitivity in the context of ultrasonic nondestructive testing. Engineering Analysis with Boundary Elements, 32:936-947.

[Amstutz et al., 2005] Amstutz, S., Horchani, I., and Masmoudi, M. (2005). Crack detection by the topological gradient method. Control Cybernet., 34(1):81-101. 
[Amstutz et al., 2012] Amstutz, S., Novotny, A. A., and de Souza Neto, E. A. (2012). Topological derivative-based topology optimization of structures subject to Drucker-Prager stress constraints. Comput. Methods Appl. Mech. Engrg., 233/236:123-136.

[Amstutz et al., 2014] Amstutz, S., Novotny, A. A., and Van Goethem, N. (2014). Topological sensitivity analysis for elliptic differential operators of order 2m. J. Differential Equations, 256(4):1735-1770.

[Amstutz et al., 2008] Amstutz, S., Takahashi, T., and Vexler, B. (2008). Topological sensitivity analysis for time-dependent problems. ESAIM Control Optim. Calc. Var., 14(3):427-455.

[Attouch et al., 2014] Attouch, H., Buttazzo, G., and Michaille, G. (2014). Variational analysis in Sobolev and BV spaces, volume 17 of MOS-SIAM Series on Optimization. Second edition.

[Aubert and Drogoul, 2015] Aubert, G. and Drogoul, A. (2015). Topological gradient for a fourth order operator used in image analysis. ESAIM Control Optim. Calc. Var., 21(4):1120-1149.

[Bellis and Bonnet, 2013] Bellis, C. and Bonnet, M. (2013). Qualitative identification of cracks using 3D transient elastodynamic topological derivative: formulation and FE implementation. Comput. Methods Appl. Mech. Engrg., 253:89-105.

[Ben Abda et al., 0910] Ben Abda, A., Hassine, M., Jaoua, M., and Masmoudi, M. (2009/10). Topological sensitivity analysis for the location of small cavities in Stokes flow. SIAM J. Control Optim., 48(5):2871-2900.

[Bonnet, 2006] Bonnet, M. (2006). Topological sensitivity for 3D elastodynamic and acoustic inverse scattering in the time domain. Comput. Methods Appl. Mech. Engrg., 195(37-40):5239-5254.

[Bonnet, 2009] Bonnet, M. (2009). Higher-order topological sensitivity for 2-D potential problems. Application to fast identification of inclusions. Internat. J. Solids Structures, 46(11-12):2275-2292.

[Bonnet, 2011] Bonnet, M. (2011). Fast identification of cracks using higher-order topological sensitivity for 2-D potential problems. Eng. Anal. Bound. Elem., 35(2):223-235.

[Bonnet and Cornaggia, 2017] Bonnet, M. and Cornaggia, R. (2017). Higher order topological derivatives for three-dimensional anisotropic elasticity. ESAIM Math. Model. Numer. Anal., 51(6):20692092 .

[Bonnet and Delgado, 2013] Bonnet, M. and Delgado, G. (2013). The topological derivative in anisotropic elasticity. Quart. J. Mech. Appl. Math., 66(4):557-586.

[Brezis, 2011] Brezis, H. (2011). Functional analysis, Sobolev spaces and partial differential equations. Universitext. Springer, New York.

[Dautray and Lions, 1988] Dautray, R. and Lions, J.-L. (1988). Analyse mathématique et calcul numérique pour les sciences et les techniques. Vol. 4. Masson, Paris.

[Delfour and Sturm, 2017] Delfour, M. C. and Sturm, K. (2017). Parametric semidifferentiability of minimax of Lagrangians: averaged adjoint approach. J. Convex Anal., 24(4):1117-1142.

[Demengel and Demengel, 2012] Demengel, F. and Demengel, G. (2012). Functional spaces for the theory of elliptic partial differential equations. Universitext. Springer, London; EDP Sciences, Les Ulis. Translated from the 2007 French original by Reinie Erné. 
[Deny and Lions, 1955] Deny, J. and Lions, J. L. (1955). Les espaces du type de Beppo Levi. Ann. Inst. Fourier (Grenoble), 5:305-370.

[Eschenauer et al., 1994] Eschenauer, H., Kobelev, V., and Schumacher, A. (1994). Bubble method for topology and shape optimization of structures. Structural optimization, 8:42-51.

[Gangl and Sturm, 2020] Gangl, P. and Sturm, K. (2020). A simplified derivation technique of topological derivatives for quasi-linear transmission problems. ESAIM Control Optim. Calc. Var., 26:Paper No. 106, 20.

[Garreau et al., 2001] Garreau, S., Guillaume, P., and Masmoudi, M. (2001). The topological asymptotic for PDE systems: the elasticity case. SIAM J. Control Optim., 39(6):1756-1778.

[Guillaume and Sid Idris, 2002] Guillaume, P. and Sid Idris, K. (2002). The topological asymptotic expansion for the Dirichlet problem. SIAM J. Control Optim., 41(4):1042-1072.

[Guillaume and Sid Idris, 2004] Guillaume, P. and Sid Idris, K. (2004). Topological sensitivity and shape optimization for the Stokes equations. SIAM J. Control Optim., 43(1):1-31.

[Guzina and Bonnet, 2006] Guzina, B. B. and Bonnet, M. (2006). Small-inclusion asymptotic of misfit functionals for inverse problems in acoustics. Inverse Problems, 22(5):1761-1785.

[Hassine et al., 2007] Hassine, M., Jan, S., and Masmoudi, M. (2007). From differential calculus to 0-1 topological optimization. SIAM J. Control Optim., 45(6):1965-1987.

[Henrot and Pierre, 2018] Henrot, A. and Pierre, M. (2018). Shape variation and optimization, volume 28 of EMS Tracts in Mathematics. European Mathematical Society (EMS), Zürich.

[Hintermüller et al., 2012] Hintermüller, M., Laurain, A., and Novotny, A. A. (2012). Second-order topological expansion for electrical impedance tomography. Adv. Comput. Math., 36(2):235-265.

[Iguernane et al., 2009] Iguernane, M., Nazarov, S. A., Roche, J.-R., Sokolowski, J., and Szulc, K. (2009). Topological derivatives for semilinear elliptic equations. Int. J. Appl. Math. Comput. Sci., 19(2):191-205.

[Masmoudi, 2002] Masmoudi, M. (2002). The topological asymptotic. In Glowinski, R., Karawada, H., and Periaux, J., editors, Computational Methods for Control Applications, pages 53-72. GAKUTO International Series.

[Masmoudi et al., 2005] Masmoudi, M., Pommier, J., and Samet, B. (2005). The topological asymptotic expansion for the Maxwell equations and some applications. Inverse Problems, 21(2):547-564.

[Murat and Simon, 1976] Murat, F. and Simon, J. (1976). Sur le contrôle par un domaine géométrique. Technical Report 76 015, L. d'Analyse Numérique Univ. Paris 6.

[Novotny, 2003] Novotny, A.-A. (2003). Análise de sensibilidade topológica. PhD thesis, Laboratório Nacional de Computação Científica, Petrópolis, Brazil.

[Novotny and Sokolowski, 2013] Novotny, A. A. and Sokolowski, J. (2013). Topological derivatives in shape optimization. Interaction of Mechanics and Mathematics. Springer, Heidelberg.

[Novotny and Sokolowski, 2020] Novotny, A. A. and Sokolowski, J. (2020). An introduction to the topological derivative method. Springer. 
[Novotny et al., 2019a] Novotny, A. A., Sokolowski, J., and Żochowski, A. (2019a). Topological derivatives of shape functionals. Part I: Theory in singularly perturbed geometrical domains. J. Optim. Theory Appl., 180(2):341-373.

[Novotny et al., 2019b] Novotny, A. A., Sokolowski, J., and Żochowski, A. (2019b). Topological derivatives of shape functionals. Part III: Second-order method and applications. J. Optim. Theory Appl., 181(1):1-22.

[Ortner and Süli, 2012] Ortner, C. and Süli, E. (2012). A note on linear elliptic systems on $\mathbb{R}^{d}$. Technical Report 1202:3970, ArXiv.

[Pólya and Szegö, 1951] Pólya, G. and Szegö, G. (1951). Isoperimetric Inequalities in Mathematical Physics. Annals of Mathematics Studies, No. 27. Princeton University Press, Princeton, N. J.

[Samet et al., 2003] Samet, B., Amstutz, S., and Masmoudi, M. (2003). The topological asymptotic for the Helmholtz equation. SIAM J. Control Optim., 42(5):1523-1544.

[Schiffer and Szegö, 1949] Schiffer, M. and Szegö, G. (1949). Virtual mass and polarization. Trans. Amer. Math. Soc., 67:130-205.

[Schumacher, 1995] Schumacher, A. (1995). Topologieoptimisierung von Bauteilstrukturen unter Verwendung von Lopchpo- sitionierungkrieterien. PhD thesis, Universität-Gesamthochschule-Siegen.

[Sokolowski and Żochowski, 1999] Sokolowski, J. and Żochowski, A. (1999). On the topological derivative in shape optimization. SIAM J. Control Optim., 37(4):1251-1272.

[Sokolowski and Zolésio, 1992] Sokolowski, J. and Zolésio, J.-P. (1992). Introduction to shape optimization, volume 16 of Springer Series in Computational Mathematics. Springer-Verlag, Berlin. Shape sensitivity analysis.

[Sturm, 2020] Sturm, K. (2020). Topological sensitivities via a Lagrangian approach for semilinear problems. Nonlinearity, 33(9):4310-4337. 